\title{
Quantum-mechanical reaction rate constants from centroid molecular dynamics simulations
}

\author{
Eitan Geva ${ }^{\text {a) }}$ and Qiang Shi \\ Department of Chemistry, University of Michigan, Ann Arbor, Michigan 48109-1055 \\ Gregory A. Voth \\ Department of Chemistry and Henry Eyring Center for Theoretical Chemistry, University of Utah, \\ Salt Lake City, Utah 84112-0850
}

(Received 17 July 2001; accepted 30 August 2001)

\begin{abstract}
It has been shown recently that in order for real-time correlation functions obtained from centroid molecular dynamics (CMD) simulations to be directly related, without further approximations, to the corresponding quantum correlation functions, one of the operators should be linear in the position and/or momentum [Jang and Voth, J. Chem. Phys. 111, 2357 (1999)]. Standard reaction rate theory relates the rate constant to the flux-Heaviside or the flux-flux correlation functions, which involve two nonlinear operators and therefore cannot be calculated via CMD without further approximations. We present an alternative, and completely equivalent, reaction rate theory which is based on the position-flux correlation function. The new formalism opens the door to more rigorously using CMD for the calculation of quantum reaction rate constants in general many-body systems. The new method is tested on a system consisting of a double-well potential bilinearly coupled to a harmonic bath. The results obtained via CMD are found to be in good agreement with the numerically exact results for a wide range of frictions and temperatures. (C) 2001 American Institute of Physics. [DOI: 10.1063/1.1412870]
\end{abstract}

\section{INTRODUCTION}

The effect of dissipative environments on barrier crossing rates has been a central concern of theoretical chemistry for many decades. ${ }^{1}$ Until quite recently, most computations of reaction rates in condensed matter were based on classical mechanics. Classical calculations are rather accurate at high temperature. However, significant quantum corrections to the classical rate emerge at intermediate temperatures. At even lower temperatures, below a characteristic crossover temperature, quantum tunneling becomes the dominant mechanism and the rate becomes weakly dependent on temperature. Quantum effects play a particularly important role when light particles such as protons and electrons are involved. In general, the quantum reaction rate is enhanced relative to the classical rate, due to tunneling and zero-point energy effects. The coupling to a dissipative environment is generally found to diminish this quantum enhancement, and causes other interesting effects such as the "Kramers turnover." 1

Several attempts to evaluate solution-phase quantum reaction rate constants have been made. Early attempts originated from efforts to develop a "quantum transition state theory" (QTST). Of these, one of the the most successful is Path-integral-QTST (PI-QTST), ${ }^{2-6}$ which is based on the centroid concept. ${ }^{2,7,8}$ This concept comes from the pathintegral formulation of quantum mechanics, ${ }^{9,10}$ according to which the equilibrium thermodynamics of a quantum particle is analogous to that of a classical cyclic chain of beads connected by harmonic springs. ${ }^{11,12}$ The center of mass of such a

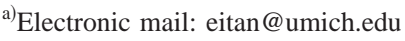

chain is known as its centroid. The structure of PI-QTST is similar to that of classical TST, ${ }^{13}$ except that the classical position is replaced by the centroid of the corresponding chain.

Attempts to go beyond QTST were based mostly on an expression independently derived by Yamamoto ${ }^{14}$ and by Miller and co-workers, ${ }^{15,16}$ which relates the reaction rate constant with the equilibrium flux autocorrelation function. Makri and co-workers have presented a numerical procedure for evaluating the quantum flux autocorrelation function, and hence the exact quantum rate constant, for a reaction that takes place along a well-defined reaction coordinate, in a harmonic bath. ${ }^{17,18}$ This methodology is based on the influence functional formalism of quantum dissipative dynamics, ${ }^{19-21}$ and involves the evaluation of real-time Feynman path integrals. ${ }^{9}$ The restriction to a harmonic bath arises from the fact that, in this case, the influence functional can be evaluated analytically. Such an analytical solution is not available when the bath is anharmonic. Unfortunately, the numerical calculation of real-time path integrals is notoriously difficult due to the infamous sign problem, although recent attempts based on semiclassical approximations are quite promising. ${ }^{18,22-26}$

An alternative approach is based on analytical continuation of imaginary time flux autocorrelation functions. ${ }^{27-29}$ Imaginary-time path integrals can be calculated numerically for relatively complex systems, by performing Monte Carlo or molecular dynamics simulations on the corresponding classical chains (PIMC and PIMD, respectively). ${ }^{11,12}$ However, difficulties arise due to the fact that transforming from imaginary time to real time is numerically unstable. Never- 
theless, Berne and co-workers have recently shown that some of these difficulties may be resolved, at least at intermediate temperatures, by using a maximum entropy procedure $^{30-32,28}$ and by incorporating knowledge on the short-time dynamics. ${ }^{33,29}$ It still remains to be seen if this approach will also be applicable under low temperature conditions, and in the case of more anharmonic systems.

In the present paper, we introduce a new and rather different approach to the calculation of quantum reaction rate constants. Like PI-QTST, it is based on the centroid concept. However, it avoids any kind of TST-like approximations, and explicitly accounts for dynamical effects within the framework of centroid molecular dynamics (CMD). CMD is an approximate method for calculating real-time quantum correlation functions. ${ }^{5,34-40}$ It is based on the hypothesis that the centroid follows classical-like dynamics, and that quantum effects can be incorporated by modifying the initial sampling and the force fields, as well as by representing dynamical observables by suitably defined "centroid symbols." In the last few years, CMD has been shown to be useful and computationally feasible for realistic, complex, many-body systems (see, e.g., Refs. 41-50).

The main obstacle that stands in the way of calculating reaction rate constants via CMD has to do with the following fact: In order for real-time correlation functions obtained from CMD simulations to be directly related to the corresponding quantum correlation functions, one of the operators must be linear in the position and/or momentum. ${ }^{39}$ The flux autocorrelation function does not satisfy this criterion, due to the nonlinear nature of the flux operator. As a result, previous attempts to use CMD for calculating the flux autocorrelation function had to involve additional approximations whose validity is not always clear. ${ }^{35,36,51}$ In contrast, the new method presented below avoids any approximations other than those which are intrinsic to CMD. ${ }^{39,40}$ This is made possible by starting with a different, yet completely equivalent, expression for the reaction rate constant, which involves another correlation function that is linear in the position and as such can be calculated directly via CMD.

The remainder of this paper is organized in the following way: A short overview of CMD for a many-body system is given in Sec. II. The new method for calculating reaction rate constants is then discussed in Sec. III, while the method is tested on a system consisting of a double-well potential bilinearly coupled to a harmonic bath in Sec. IV, where a comparison to the exact results is also made. The main conclusions of this work are summarized in Sec. V.

\section{CENTROID MOLECULAR DYNAMICS (CMD)}

In this section, we give a short overview of CMD, in the context of a many-body system, with emphasis on results that are important and useful for the present study. The discussion follows Refs. 38 and 39, where further details may be found.

Consider a general quantum system with $N$ degrees of freedom whose quantum mechanical Hamiltonian operator is

$$
\hat{H}=\sum_{i=1}^{N} \frac{\left(\hat{p}^{(i)}\right)^{2}}{2 m^{(i)}}+V\left(\hat{x}^{(1)}, \ldots, \hat{x}^{(N)}\right) \equiv \sum_{i=1}^{N} \frac{\left(\hat{p}^{(i)}\right)^{2}}{2 m^{(i)}}+V(\hat{\mathbf{x}}) .
$$

Here, as in the rest of this paper, we use boldface letters for vectors and letters capped with a "hat," e.g. $\hat{A}$, for operators. $\hat{\mathbf{x}}=\left(\hat{x}^{(1)}, \ldots, \hat{x}^{(N)}\right)$ and $\hat{\mathbf{p}}=\left(\hat{p}^{(1)}, \ldots, \hat{p}^{(N)}\right)$ are the vector operators that represent the Cartesian coordinates and conjugate momenta, and $\left\{m^{(i)}\right\}$ are the corresponding masses. Canonical equilibrium is described by the following normalized density operator:

$$
\hat{\rho}=e^{-\beta \hat{H}} / Z,
$$

where $Z=\operatorname{Tr}\left(e^{-\beta \hat{H}}\right)$. The equilibrium expectation value of an observable, $\hat{A}$, is given by

$$
\langle\hat{A}\rangle=\operatorname{Tr}(\hat{\rho} \hat{A}) .
$$

CMD is based on an alternative representation of Eqs. (2) and (3) in terms of a classical-like phase-space description, ${ }^{39,40}$

$$
\langle\hat{A}\rangle \equiv\left\langle A_{c}\right\rangle_{c}=\frac{1}{Z} \iint \frac{d \mathbf{x}_{\mathbf{c}} d \mathbf{p}_{\mathbf{c}}}{(2 \pi \hbar)^{N}} \rho_{c}\left(\mathbf{x}_{\mathbf{c}}, \mathbf{p}_{\mathbf{c}}\right) A_{c}\left(\mathbf{x}_{\mathbf{c}}, \mathbf{p}_{\mathbf{c}}\right),
$$

where $\mathbf{x}_{\mathbf{c}}=\left(x_{c}^{(1)}, \ldots, x_{c}^{(N)}\right) \quad$ and $\quad \mathbf{p}_{\mathbf{c}}=\left(p_{c}^{(1)}, \ldots, p_{c}^{(N)}\right)$ are classical-like coordinates and momenta, and

$$
Z \equiv \iint \frac{d \mathbf{x}_{\mathbf{c}} d \mathbf{p}_{\mathbf{c}}}{(2 \pi \hbar)^{N}} \rho_{c}\left(\mathbf{x}_{\mathbf{c}}, \mathbf{p}_{\mathbf{c}}\right) .
$$

As is well known, the transformation between the quantum operators, $\hat{\rho}$ and $\hat{A}$, and the corresponding phase-space functions, $\rho_{c}\left(\mathbf{x}_{\mathbf{c}}, \mathbf{p}_{\mathbf{c}}\right)$ and $A_{c}\left(\mathbf{x}_{\mathbf{c}}, \mathbf{p}_{\mathbf{c}}\right)$, is not unique. For example, the Wigner and Husimi transforms provide two such distinctly different phase-space representations. ${ }^{52}$ The centroid formalism is based on yet another choice of such a phasespace representation. In this case, the classical-like phasespace probability density is given by the following "centroid probability density:"

$$
\rho_{c}\left(\mathbf{x}_{\mathbf{c}}, \mathbf{p}_{\mathbf{c}}\right)=\rho_{c}\left(\mathbf{x}_{\mathbf{c}}\right) \exp \left[-\beta \sum_{i=1}^{N} \frac{\left(p_{c}^{(i)}\right)^{2}}{2 m^{(i)}}\right],
$$

where

$$
\begin{aligned}
\rho_{c}\left(\mathbf{x}_{\mathbf{c}}\right)= & \prod_{i=1}^{N}\left(\frac{2 \pi \beta \hbar^{2}}{m^{(i)}}\right)^{1 / 2} \int_{\mathbf{x}(0)=\mathbf{x}(\beta \hbar)} D \mathbf{x}(\tau) \delta\left[\mathbf{x}_{\mathbf{c}}\right. \\
& \left.-(\beta \hbar)^{-1} \int_{0}^{\beta \hbar} d \tau \mathbf{x}(\tau)\right] \exp \{-\mathcal{S}[\mathbf{x}(\tau)] / \hbar\} \\
= & \lim _{P \rightarrow \infty} \prod_{i=1}^{N}\left\{\left(\frac{2 \pi \beta \hbar^{2}}{m^{(i)}}\right)^{1 / 2}\left(\frac{m^{(i)} P}{2 \pi \beta \hbar^{2}}\right)^{P / 2}\right\} \\
& \times \int d \mathbf{x}_{\mathbf{1}} \cdots \int d \mathbf{x}_{\mathbf{P}} \delta\left(\mathbf{x}_{\mathbf{c}}-\frac{1}{P} \sum_{k=1}^{P} \mathbf{x}_{\mathbf{k}}\right) \\
& \times \exp \left\{-\mathcal{S}\left[\mathbf{x}_{\mathbf{1}}, \ldots, \mathbf{x}_{\mathbf{P}}\right] / \hbar\right\}
\end{aligned}
$$

with 


$$
\begin{aligned}
\frac{1}{\hbar} \mathcal{S}[\mathbf{x}(\tau)] & =\lim _{P \rightarrow \infty} \frac{1}{\hbar} \mathcal{S}\left[\mathbf{x}_{\mathbf{1}}, \ldots, \mathbf{x}_{\mathbf{P}}\right] \\
& =\frac{1}{\hbar} \int_{0}^{\beta \hbar} d \tau\left[\sum_{i=1}^{N} \frac{1}{2} m^{(i)}\left[\dot{x}^{(i)}(\tau)\right]^{2}+V(\mathbf{x}(\tau))\right],
\end{aligned}
$$

and

$$
\begin{aligned}
\frac{1}{\hbar} \mathcal{S}\left[\mathbf{x}_{\mathbf{1}}, \ldots, \mathbf{x}_{\mathbf{P}}\right]= & \beta\left\{\sum_{i=1}^{N} \sum_{k=1}^{P} \frac{1}{2} m^{(i)} \omega_{P}^{2}\left(x_{k}^{(i)}-x_{k+1}^{(i)}\right)^{2}\right. \\
& \left.-\frac{1}{P} \sum_{k=1}^{P} V\left(\mathbf{x}_{\mathbf{k}}\right)\right\} .
\end{aligned}
$$

In Eq. (9), $\mathbf{x}_{\mathbf{P}+\mathbf{1}}=\mathbf{x}_{\mathbf{1}}$ and $\omega_{p}^{2}=P /(\beta \hbar)^{2}$.

Two important points should be noted with respect to Eqs. (6)-(9)

(1) $\rho_{c}\left(\mathbf{x}_{\mathbf{c}}\right)$ is given by an imaginary time path integral over all possible cyclic paths, and is proportional to the probability density of finding a classical system consisting of $N$ cyclic chains with their centers of mass (the centroids) at $x_{c}^{(1)}, \ldots, x_{c}^{(N)}$, i.e., at $\mathbf{x}=\mathbf{x}_{\mathbf{c}}$.

(2) Unlike other phase-space representations of the canonical quantum density operator, $\rho_{c}\left(\mathbf{x}_{\mathbf{c}}, \mathbf{p}_{\mathbf{c}}\right)$ assumes a classical-like form. This is made clear by defining a centroid potential, $V_{c m}\left(\mathbf{x}_{\mathbf{c}}\right)$, such that

$\rho_{c}\left(\mathbf{x}_{\mathbf{c}}\right) \equiv e^{-\beta V_{c m}\left(\mathbf{x}_{\mathbf{c}}\right)}$.

[It is important to note that $V_{c m}\left(\mathbf{x}_{\mathbf{c}}\right)$ is generally different than the centroid symbol of the potential operator. $\left.{ }^{39,40}\right]$

The corresponding transformation between $\hat{A}$ and its centroid symbol, $A_{c}$, which ensures the validity of Eq. (4), is given by ${ }^{39}$

$$
A_{c}\left(\mathbf{x}_{\mathbf{c}}, \mathbf{p}_{\mathbf{c}}\right)=\operatorname{Tr}\left\{\hat{\delta}_{c}\left(\mathbf{x}_{\mathbf{c}}, \mathbf{p}_{\mathbf{c}}\right) \hat{A}\right\}
$$

where $\hat{\delta}_{c}\left(\mathbf{x}_{\mathbf{c}}, \mathbf{p}_{\mathbf{c}}\right)$, which has been denoted the quasi-density operator, and whose trace is equal to 1 , is given by

$$
\hat{\delta}_{c}\left(\mathbf{x}_{\mathbf{c}}, \mathbf{p}_{\mathbf{c}}\right)=\frac{\hat{\varphi}\left(\mathbf{x}_{\mathbf{c}}, \mathbf{p}_{\mathbf{c}}\right)}{\rho_{c}\left(\mathbf{x}_{\mathbf{c}}, \mathbf{p}_{\mathbf{c}}\right)}
$$

with

$$
\hat{\varphi}\left(\mathbf{x}_{\mathbf{c}}, \mathbf{p}_{\mathbf{c}}\right)=\left(\frac{\hbar}{2 \pi}\right)^{N} \int_{-\infty}^{\infty} \mathbf{d} \zeta \int_{-\infty}^{\infty} \mathbf{d} \boldsymbol{\eta} e^{\mathbf{i} \zeta \cdot\left(\hat{\mathbf{x}}-\mathbf{x}_{\mathbf{c}}\right)+\mathbf{i} \boldsymbol{\eta} \cdot\left(\hat{\mathbf{p}}-\mathbf{p}_{\mathbf{c}}\right)-\beta \hat{H}}
$$

$\left[\boldsymbol{\zeta}=\left(\zeta^{(1)}, \ldots, \zeta^{(N)}\right), \quad \boldsymbol{\eta}=\left(\eta^{(1)}, \ldots, \eta^{(N)}\right)\right]$. In practice, it is convenient to use the path integral representation of $\hat{\varphi}\left(\mathbf{x}_{\mathbf{c}}, \mathbf{p}_{\mathbf{c}}\right)$ in order to find the centroid symbol of a given quantum observable. ${ }^{39}$ In particular, the centroid position and momentum can be shown to coincide with the centroid symbols of the position and momentum operators. ${ }^{39,40}$

$$
\mathbf{x}_{\mathbf{c}}=\operatorname{Tr}\left\{\hat{\delta}_{c}\left(\mathbf{x}_{\mathbf{c}}, \mathbf{p}_{\mathbf{c}}\right) \hat{\mathbf{x}}\right\}, \quad \mathbf{p}_{\mathbf{c}}=\operatorname{Tr}\left\{\hat{\delta}_{c}\left(\mathbf{x}_{\mathbf{c}}, \mathbf{p}_{\mathbf{c}}\right) \hat{\mathbf{p}}\right\}
$$

CMD is a method for calculating real-time equilibrium correlation functions in many-body quantum systems. Starting with the initial state $\left(\mathbf{x}_{\mathbf{c}}, \mathbf{p}_{\mathbf{c}}\right)$, the dynamics of a centroid observables, $A_{c}$, is exactly given by ${ }^{39}$

$$
A_{c}\left(t ; \mathbf{x}_{\mathbf{c}}, \mathbf{p}_{\mathbf{c}}\right)=\operatorname{Tr}\left\{\hat{\delta}_{c}\left(t ; \mathbf{x}_{\mathbf{c}}, \mathbf{p}_{\mathbf{c}}\right) \hat{A}\right\}
$$

where,

$$
\hat{\delta}_{c}\left(t ; \mathbf{x}_{\mathbf{c}}, \mathbf{p}_{\mathbf{c}}\right)=e^{-i \hat{H} t / \hbar} \hat{\delta}_{c}\left(\mathbf{x}_{\mathbf{c}}, \mathbf{p}_{\mathbf{c}}\right) e^{i \hat{H} t / \hbar}
$$

In particular, the dynamics of the centroid positions and momenta are governed by the following classical-like equations of motion:

$$
\begin{aligned}
& \frac{d}{d t} x_{c}^{(i)}(t)=\frac{p_{c}^{(i)}(t)}{m^{(i)}}, \\
& \frac{d}{d t} p_{c}^{(i)}(t)=f_{c}^{(i)}\left(t ; \mathbf{x}_{\mathbf{c}}\right) .
\end{aligned}
$$

Here, $\mathbf{f}_{\mathbf{c}}\left(t=0 ; \mathbf{x}_{\mathbf{c}}\right) \equiv \mathbf{f}_{\mathbf{c}}\left(\mathbf{x}_{\mathbf{c}}\right)$ is the centroid symbol of the force, which can be shown to satisfy the following rather remarkable identity: ${ }^{39}$

$$
\mathbf{f}_{\mathbf{c}}\left(\mathbf{x}_{\mathbf{c}}\right)=\operatorname{Tr}\left\{\hat{\delta}_{c}\left(\mathbf{x}_{\mathbf{c}}, \mathbf{p}_{\mathbf{c}}\right)\left[-\boldsymbol{\nabla}_{\mathbf{x}} V(\hat{\mathbf{x}})\right]\right\}=-\boldsymbol{\nabla}_{\mathbf{x}_{\mathbf{c}}} V_{c m}\left(\mathbf{x}_{\mathbf{c}}\right)
$$

It should be emphasized that despite the classical-like appearance of Eqs. (17) and (18), the dynamics obtained by exactly solving them is fully quantum mechanical [cf. Eq. (16)].

CMD is based on the following approximation for the quantum dynamics of the centroid symbol, $A_{c}$, of any dynamical observable $\hat{A}:{ }^{40}$

$$
A_{c}\left[t ; \mathbf{x}_{\mathbf{c}}, \mathbf{p}_{\mathbf{c}}\right] \approx A_{c}\left[\mathbf{x}_{\mathbf{c}}(t), \mathbf{p}_{\mathbf{c}}(t)\right]
$$

In particular, applying this approximation to the centroid symbol of the force yields

$$
\mathbf{f}_{\mathbf{c}}\left[t ; \mathbf{x}_{\mathbf{c}}, \mathbf{p}_{\mathbf{c}}\right] \approx \mathbf{f}_{\mathbf{c}}\left[\mathbf{x}_{\mathbf{c}}(t), \mathbf{p}_{\mathbf{c}}(t)\right]
$$

which gives the equations of motion, Eqs. (17)-(18), a closed classical-like structure. The approximation in Eq. (20) becomes exact in one, or all, of three cases: (1) when the time goes to zero; (2) in the classical limit; and (3) in harmonic systems. However, a variety of examples has demonstrated that this approximation is in fact useful for a wide range of realistic systems, especially for short times. The strength of the CMD approximation comes from the fact that it reduces quantum dynamics into classical dynamics on the centroid potential surface, which can be obtained from computationally feasible imaginary-time path-integral simulations. Furthermore, at long times, CMD reproduces the exact equilibrium centroid distribution, and hence the exact regression behavior limit. ${ }^{40}$

The relationship between correlation functions directly calculated from CMD simulations and the corresponding quantum equilibrium correlation functions is based on the following identity: ${ }^{39,51}$ 


$$
\begin{aligned}
& \frac{1}{\beta} \int_{0}^{\beta} d \lambda e^{-(\beta-\lambda) \hat{H}} \hat{\mathbf{x}} e^{-\lambda \hat{H}} \\
& \quad=\iint \frac{d \mathbf{x}_{\mathbf{c}} d \mathbf{p}_{\mathbf{c}}}{(2 \pi \hbar)^{N}} \rho_{c}\left(\mathbf{x}_{\mathbf{c}}, \mathbf{p}_{\mathbf{c}}\right) \mathbf{x}_{\mathbf{c}} \hat{\delta}_{c}\left(\mathbf{x}_{\mathbf{c}}, \mathbf{p}_{\mathbf{c}}\right) .
\end{aligned}
$$

Multiplying Eq. (22) by $e^{i \hat{H} t / \hbar} \hat{B} e^{-i \hat{H} t / \hbar}$, where $\hat{B}$ is any operator, and tracing over the result, leads to the following identity:

$$
\frac{1}{Z} \iint \frac{d \mathbf{x}_{\mathbf{c}} d \mathbf{p}_{\mathbf{c}}}{(2 \pi \hbar)^{N}} \rho_{c}\left(\mathbf{x}_{\mathbf{c}}, \mathbf{p}_{\mathbf{c}}\right) \mathbf{x}_{\mathbf{c}} B_{c}\left(t ; \mathbf{x}_{\mathbf{c}}, \mathbf{p}_{\mathbf{c}}\right)=C_{\hat{\mathbf{x}} \hat{B}}^{\text {Kubo }}(t),
$$

where

$$
\begin{aligned}
C_{\hat{\mathbf{x}} \hat{B}}^{\text {Kubo }}(t) & =\frac{1}{\beta Z} \int_{0}^{\beta} d \lambda \operatorname{Tr}\left\{e^{-(\beta-\lambda) \hat{H}} \hat{\mathbf{x}} e^{-\lambda \hat{H}} e^{i \hat{H} t / \hbar} \hat{B} e^{-i \hat{H} t / \hbar}\right\} \\
& =\frac{1}{\beta} \int_{0}^{\beta} d \lambda\langle\hat{\mathbf{x}}(0) \hat{B}(t+i \hbar \lambda)\rangle
\end{aligned}
$$

is the exact quantum Kubo-transformed correlation function. ${ }^{53}$ Note that the following definition of the "regular" quantum correlation function was utilized in the last equality of Eq. (24):

$$
C_{\hat{A}, \hat{B}}(t)=\langle\hat{A}(0) \hat{B}(t)\rangle=\frac{1}{Z} \operatorname{Tr}\left\{e^{-\beta \hat{H}} \hat{A} e^{i \hat{H} t / \hbar} \hat{B} e^{-i \hat{H} t / \hbar}\right\} .
$$

Applying the CMD approximation, Eq. (20), to $B_{c}\left(t ; \mathbf{x}_{\mathbf{c}}, \mathbf{p}_{\mathbf{c}}\right)$ in Eq. (23), leads to the following approximate expression for this quantum correlation function:

$$
C_{\hat{\mathbf{x}} \hat{B}}^{\text {Kubo }}(t) \approx \frac{1}{Z} \iint \frac{d \mathbf{x}_{\mathbf{c}} d \mathbf{p}_{\mathbf{c}}}{(2 \pi \hbar)^{N}} \rho_{c}\left(\mathbf{x}_{\mathbf{c}}, \mathbf{p}_{\mathbf{c}}\right) \mathbf{x}_{\mathbf{c}} B_{c}\left[\mathbf{x}_{\mathbf{c}}(t), \mathbf{p}_{\mathbf{c}}(t)\right] .
$$

An important point is that Eq. (23) still holds if $\hat{\mathbf{x}}$ and $\mathbf{x}_{\mathbf{c}}$ are substituted by $\hat{\mathbf{p}}$ and $\mathbf{p}_{\mathbf{c}}$, respectively, or by a linear combination of these two operators, but does not hold for other types of nonlinear operators. Hence, the relationship between correlation functions that are calculated from CMD and the corresponding quantum correlation functions is only well established when one of the operators is linear in $\hat{\mathbf{x}}$ and/or $\hat{\mathbf{p}}$.

Several attempts have been made to extend the applicability of CMD to correlation functions involving two nonlinear operators. ${ }^{35,43,51,54}$ In practice, all of these approaches involved additional approximations. In this paper we pursue an alternative approach, where instead of trying to change the CMD formalism so that it can be used for directly calculating correlation functions with two nonlinear operators, we express the desired quantity in terms of a correlation function which has the form of that in Eq. (24). This approach is demonstrated in the following section for the case of the reaction rate constant.

\section{REACTION RATE THEORY}

In this section we discuss the quantum theory of reaction rate constants. We start by defining the phenomenological kinetics upon which the concept of the reaction rate constant is based. This is followed by a discussion on the calculation of reaction rate constants via linear-response theory. The section closes with a discussion of how reaction rate constants can be calculated directly from CMD simulations, without additional approximations to the correlation functions.

\section{A. Phenomenology}

We will focus, for simplicity, on a unimolecular reaction, such as isomerization, that takes place in solution, along a predefined reaction coordinate. The total Hamiltonian is given by

$$
\hat{H}=\frac{\hat{p}^{2}}{2 m}+\sum_{i=1}^{N} \frac{\left(\hat{P}^{(i)}\right)^{2}}{2 M^{(i)}}+\widetilde{V}_{0}\left(\hat{s}^{\prime}\right)+V(\hat{\mathbf{Q}})+\widetilde{W}\left(\hat{\mathbf{Q}}, \hat{s}^{\prime}\right) .
$$

Here, $\hat{s}^{\prime}, \hat{p}$, and $m$ are the reaction coordinate, conjugate momentum, and corresponding mass, respectively; $\hat{\mathbf{Q}}$ $=\left(\hat{Q}^{(1)}, \ldots, \hat{Q}^{(N)}\right), \hat{\mathbf{P}}=\left(\hat{P}^{(1)}, \ldots, \hat{P}^{(N)}\right)$, and $\left\{M^{(i)}\right\}$ are the coordinates, conjugate momenta, and masses of the bath degrees of freedom, respectively; $\widetilde{V}_{0}\left(\hat{s}^{\prime}\right), V(\hat{\mathbf{Q}})$ and $\widetilde{W}\left(\hat{\mathbf{Q}}, \hat{s}^{\prime}\right)$ are the potential energies of the bare reaction coordinate, the bath, and their interaction, respectively. It is assumed here that $\widetilde{V}_{0}\left(\hat{s}^{\prime}\right)$ has the shape of a double well, and that the barrier top is located at $s^{\prime}=s^{\sharp}$. It is convenient to redefine the reaction coordinate such that its origin coincides with the position of the barrier top. Hence, a new reaction coordinate, $s$, is defined

$$
s=s^{\prime}-s^{\ddagger},
$$

and the Hamiltonian in Eq. (27) is rewritten in terms of it

$$
\hat{H}=\frac{\hat{p}^{2}}{2 m}+\sum_{i=1}^{N} \frac{\hat{P}_{i}^{2}}{2 M^{(i)}}+V_{0}(\hat{s})+V(\hat{\mathbf{Q}})+W(\hat{\mathbf{Q}}, \hat{s}) .
$$

Here, $\quad \widetilde{V}_{0}\left(\hat{s}^{\prime}\right)=V_{0}\left(\hat{s}^{\prime}-s^{\dagger}\right), \quad \widetilde{W}\left(\hat{\mathbf{Q}}, \hat{s}^{\prime}\right)=W\left(\hat{\mathbf{Q}}, \hat{s}^{\prime}-s^{\dagger}\right)$. It should be noted that the barrier top is now located at $s=0$.

Let $P_{R}$ and $P_{P}$ be the mole fractions of the reactant ( $s$ $<0)$ and the product $(s>0)$, respectively. $P_{R}$ and $P_{P}$ may be expressed as quantum expectation values

$$
P_{P}=1-P_{R}=\langle h(\hat{s})\rangle \equiv\langle\hat{h}\rangle,
$$

where $\hat{h} \equiv h(\hat{s})$ is the Heaviside function operator: $\left\langle s|h(\hat{s})| s^{\prime}\right\rangle=\delta\left(s-s^{\prime}\right)$ for $s>0$, and zero otherwise.

The concept of the reaction rate constant is based on the following phenomenology:

$$
\dot{P}_{P}=-\dot{P}_{R}=-k_{R P} P_{P}+k_{P R} P_{R},
$$

or equivalently

$$
\delta \dot{P}_{i}=-k \delta P_{i} .
$$

Here, $i=P$ or $R, \quad k=k_{P R}+k_{R P}, \quad \delta P_{i}=P_{i}-P_{i}^{e q}, \quad P_{P}^{e q}$ $=k_{P R} / k$, and $P_{R}^{e q}=k_{R P} / k$ (it should be noted that the mole fractions $P_{R}^{e q}$ and $P_{P}^{e q}$ are sometimes denoted by $x_{R}$ and $x_{P}$ ).

Relaxation processes are generally characterized by many relaxation times, not one. In order for reaction kinetics to be described by a single rate constant, the effective barrier energy, $E_{b}$, has to be high, i.e., $e^{-\beta E_{b} \ll 1}$. This is indeed the case in many problems of chemical interest, and it results in one relaxation time which is much longer than the rest, and 
with vanishingly small amplitudes of the other, faster, decays. ${ }^{55-57}$ Under these circumstances, the above phenomenology becomes valid after a short transient time during which the reaction does not make a significant progress. ${ }^{57,58}$

The reaction rate constant can be obtained from the following expression:

$$
k \approx k e^{-k t}=-\delta \dot{P}_{i}(t) / \delta P_{i}(0) .
$$

The approximation in Eq. (33) is justified as long as $t$ $\ll 1 / k$. We expect $k$ to be explicitly time dependent during an initial transient period, $0<t<t_{p}(\ll 1 / k)$, following which it will reach the "plateau region," where it acquires a fixed value. ${ }^{57,58}$ This fixed value corresponds to the actual phenomenological reaction rate constant. Thus,

$$
k=\lim _{t \rightarrow t_{p}} k(t),
$$

with $k(t) \equiv-\delta \dot{P}_{i}(t) / \delta P_{i}(0)$.

\section{B. Linear response theory and the standard reactive flux method}

Direct evaluation of the reaction rate constant from Eq. (33) would require explicit nonequilibrium quantum dynamical simulations. Such simulations are generally not feasible in condensed phase systems, due to the exponential scaling of the computational effort with the number of degrees of freedom. ${ }^{59}$ In fact, Eq. (33) is not very useful even if we assume that the dynamics is governed by classical mechanics. This is because barrier crossing processes follow rare event statistics, and prohibitively long classical molecular dynamics simulations would be required if good statistics on barrier crossing events is to be obtained.

Linear response theory provides an alternative route for calculating reaction rate constants, which help overcome both of the above difficulties. This approach is useful when the kinetics is exponential at all time scales of practical interest and if the rate constant is indeed a constant in the sense that it is independent of the initial state. Under such circumstances, the conditions under which the reaction rate constant is actually evaluated becomes a matter of convenience. ${ }^{14,60}$ Choosing the initial state in the vicinity of thermal equilibrium then allows us to take advantage of linear response theory ${ }^{14,53}$ In this subsection we briefly review, for completeness, the general formulation of linear response theory, followed by a more specialized adaptation to the case of barrier crossing kinetics.

Consider a quantum system with the total Hamiltonian $\hat{H}+f \hat{A}$ at $t<0$ and $\hat{H}$ at $t \geqslant 0$. Here, $f$ is a scalar coefficient, and $\hat{A}$ may be any perturbation that can shift the system from the equilibrium state $e^{-\beta \hat{H}} / \operatorname{Tr}\left(e^{-\beta \hat{H}}\right)$. At $t=0$, the system is assumed to be in thermal equilibrium with respect to the perturbed Hamiltonian

$$
\hat{\rho}(0)=e^{-\beta(\hat{H}+f \hat{A})} / \operatorname{Tr}\left[e^{-\beta(\hat{H}+f \hat{A})}\right] .
$$

In the linear response limit, Eq. (35) may be replaced by its expansion to first order in powers of $f^{61}$

$$
\hat{\rho}(0)=\frac{e^{-\beta \hat{H}}}{Z}\left\{1-f \int_{0}^{\beta} d \lambda \delta \hat{A}(-i \hbar \lambda)\right\},
$$

where $\quad Z=\operatorname{Tr}\left[e^{-\beta \hat{H}}\right], \quad \delta \hat{A}(\tau)=\hat{A}(\tau)-\langle\hat{A}\rangle_{e q}, \quad\langle\hat{A}\rangle_{e q}$ $=\operatorname{Tr}\left[e^{-\beta \hat{H}} \hat{A}\right] / Z$, and $\hat{A}(\tau)=e^{i \hat{H} \tau / \hbar} \hat{A} e^{-i \hat{H} \tau / \hbar}$. We may now propagate $\hat{\rho}(t)$ in the usual way, $\hat{\rho}(t)=e^{-i \hat{H} t / \hbar} \hat{\rho}(0) e^{i \hat{H} t / \hbar}$, which leads to

$$
\hat{\rho}(t)=\frac{e^{-\beta \hat{H}}}{Z}\left\{1-f \int_{0}^{\beta} d \lambda \delta \hat{A}(-i \hbar \lambda-t)\right\} .
$$

The expectation value of any observable, $\hat{B}$, at a later time can then be obtained as follows:

$$
\begin{aligned}
\langle\delta \hat{B}(t)\rangle=\operatorname{Tr}[\hat{\rho}(t) \delta \hat{B}] & =-f \int_{0}^{\beta} d \lambda\langle\delta \hat{A}(0) \delta \hat{B}(t+i \hbar \lambda)\rangle \\
& =-f \beta C_{\delta \hat{A}, \delta \hat{B}}^{\text {Kubo }}(t),
\end{aligned}
$$

where $C_{\delta \hat{A}, \delta \hat{B}}^{\text {Kubo }}(t)$ is a quantum Kubo-transformed correlation function [cf. Eq. (24)].

In the case of reaction kinetics, the observed quantity is the instantaneous mole fraction of the product. This dictates that $\hat{B}=h(\hat{s})$ in Eq. (38). However, we are left with almost complete freedom regarding the choice of the actual perturbation, $\hat{A}$. The standard linear response expression for the reaction rate constant corresponds to one particular choice, ${ }^{14,62}$ namely $\hat{A}=h(\hat{s})$. In this case, one substitutes $\hat{A}$ $=\hat{B}=h(\hat{s})$ in Eq. (38), to obtain

$$
\langle\delta \hat{h}(t)\rangle=-f \beta C_{\delta \hat{h}, \delta \hat{h}}^{\text {Kubo }}(t) .
$$

It can be shown that $\delta \hat{h}(t)$ commutes with $e^{-\beta \hat{H}}$ at $t \gg \hbar \beta .{ }^{63,15,16,64}$ Thus, as long as $t_{p} \gg \hbar \beta$, we may substitute the Kubo-transformed correlation function, $C_{\delta \hat{h}, \delta \hat{h}}^{\text {Kubo }}(t)$, by the regular correlation function, $C_{\delta \hat{h}, \delta \hat{h}}(t)$ [cf. Eq. (25)]:

$$
\langle\delta \hat{h}(t)\rangle=-f \beta C_{\delta \hat{h}, \delta \hat{h}}(t) .
$$

Exchanging the Kubo-transformed correlation function for a regular correlation function would usually be a welcomed simplification. However, it should be noted that this is not so in the case of $\mathrm{CMD}$, which yields correlation functions that are already in the Kubo-transformed form.

Proceeding with the above choice of $\hat{A}=h(\hat{s})$, and substituting $t=0$ in Eq. (40), we obtain

$$
\begin{aligned}
\langle\delta \hat{h}\rangle(0)=-f \beta\left\langle(\delta \hat{h})^{2}\right\rangle_{e q} & =-f \beta\langle\hat{h}\rangle_{e q}\left(1-\langle\hat{h}\rangle_{e q}\right) \\
& =-f \beta P_{P}^{e q} P_{R}^{e q} .
\end{aligned}
$$

Assuming exponential kinetics, Eq. (32), one finds that the correlation function in Eq. (39) should also decay exponentially

$$
\frac{\langle\delta \hat{h}\rangle(t)}{\langle\delta \hat{h}\rangle(0)}=\frac{C_{\delta \hat{h}, \delta \hat{h}}(t)}{P_{P}^{e q} P_{R}^{e q}}=e^{-k t} .
$$

The first equality in Eq. (42) is based on Eqs. (40) and (41), while the second equality is based on Eqs. (30) and (32). 
$\mathrm{V}_{0}(\mathrm{~s})+\mathrm{fs} \quad \mathrm{V}_{0}(\mathrm{~s})$

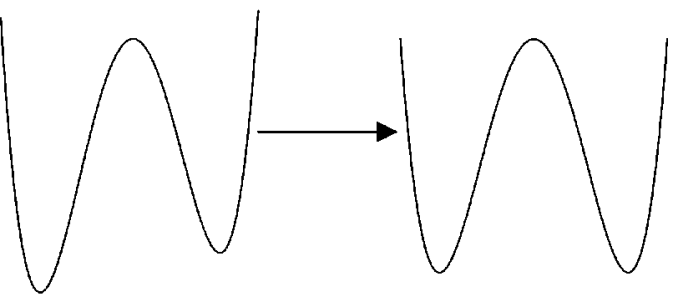

$$
\mathrm{t}<0 \quad \mathrm{t}>0
$$

FIG. 1. A schematic view of the "regression experiment." At $t<0$, the system is assumed to be equilibrated with respect to the potential energy along the reaction coordinate, $s$, which is perturbed, and the perturbation is assumed to be linear in $s$. The perturbation is removed at $t=0$, following which the system is allowed to relax to the equilibrium state with respect to its natural potential, $V_{0}(s)$. The sought-after reaction rate constant governs the relaxation of the reactant and product populations towards their new equilibrium values and is independent of the initial perturbation.

Substituting Eq. (42) into Eq. (33) yields the following set of equivalent expressions for the reaction rate constant: ${ }^{15,16}$

$$
P_{P}^{e q} P_{R}^{e q} k=-\left.\frac{d}{d t} C_{\hat{h} \hat{h}}(t)\right|_{t=t_{p}}=C_{\hat{F} \hat{h}}\left(t_{p}\right)=\int_{0}^{t_{p}} d \tau C_{\hat{F} \hat{F}}(\tau),
$$

where

$$
\hat{F}=\frac{d}{d t} \hat{h}=\frac{i}{\hbar}[\hat{H}, h(\hat{s})]=\frac{1}{2 m}[\hat{p} \delta(\hat{s})+\delta(\hat{s}) \hat{p}]
$$

is the flux operator. In deriving the first equality in Eq. (43), the correlation function $C_{\delta \hat{h}, \delta \hat{h}}(t)$ was substituted by $C_{\hat{h}, \hat{h}}(t)$ since they have the same time derivative. The second equality in Eq. (43) is based on the identity $C_{\hat{A} \dot{B}}(t)=-C_{\hat{A} \hat{B}}(t) .{ }^{65}$ The third equality in Eq. (43) is based on the identity $\operatorname{Tr}\left[e^{-\beta \hat{H}} \hat{F} \hat{h}\right]=0 .{ }^{16,64}$ The expressions in Eq. (43) are of course well known ${ }^{14-16,64}$ and widely used.
The second and third expressions for the reaction rate constant in Eq. (43) are potentially useful in practice, due to their ability to circumvent the problem of rare event statistics. The very inefficient sampling over all the initial states that do not make it to the barrier top is avoided by the spatial delta function in the flux operator, Eq. (44), which biases the sampling to initial positions at the barrier top.

\section{Reaction rate constants from CMD}

As discussed in Sec. II, using CMD to directly calculate a correlation function presently requires that at least one of the operators is linear in the coordinates and/or momenta. In the case of calculating reaction rate constants, one of the two operators is already dictated by the underlying physical observation, namely $\hat{B}=h(\hat{s})$. However, the choice of the second operator, $\hat{A}$, which corresponds to the perturbation, is essentially unconstrained in the linear response approximation. Obviously, the standard choice of $\hat{A}=h(\hat{s})$, a nonlinear operator, is not convenient if CMD is to be used, for the reasons mentioned earlier. However, we can always replace it by a more convenient choice, namely $\hat{A}=\hat{s}$, without any loss of generality. This choice will then lead to an expression for the rate constant in terms of a Kubo-transformed correlation function that can be obtained directly from CMD.

In particular, following a procedure similar to that in Sec. III B, only this time with the perturbation linear in the reaction coordinate, i.e., $\hat{A}=\hat{s}$, leads to the following expression for the reaction rate constant:

$$
k=-\frac{C_{\hat{s}, \hat{F}}^{\text {Kubo }}\left(t_{p}\right)}{C_{\delta \hat{s}, \delta \hat{h}}^{\text {Kubo }}(0)} .
$$

With the help of Eq. (23), Eq. (45) can now be put in terms of the centroid formalism

$$
k=-\frac{\int d s_{c} \int d p_{c} \int d \mathbf{Q}_{\mathbf{c}} \int d \mathbf{P}_{\mathbf{c}} \rho_{c}\left(s_{c}, p_{c}, \mathbf{Q}_{\mathbf{c}}, \mathbf{P}_{\mathbf{c}}\right) s_{c} F_{c}\left(t_{p} ; s_{c}, p_{c}, \mathbf{Q}_{\mathbf{c}}, \mathbf{P}_{\mathbf{c}}\right)}{\int d s_{c} \int d p_{c} \int d \mathbf{Q}_{\mathbf{c}} \int d \mathbf{P}_{\mathbf{c}} \rho_{c}\left(s_{c}, p_{c}, \mathbf{Q}_{\mathbf{c}}, \mathbf{P}_{\mathbf{c}}\right) \delta s_{c} \delta h_{c}\left(s_{c}, p_{c}, \mathbf{Q}_{\mathbf{c}}, \mathbf{P}_{\mathbf{c}}\right)} .
$$

The choice of the perturbation $\hat{A}=\hat{s}$ in this approach to calculate the reaction rate constant has a straightforward physical interpretation, which may not be obvious from the resulting expressions in Eqs. (45) and (46). More specifically, a linear perturbation applied along the reaction coordinate shifts the relative proportions of reactant to product. This can be seen schematically in Fig. 1, where a symmetric double well along the reaction coordinate $s$, with a barrier top at $s=0$, becomes an asymmetric double well when the perturbation $f \hat{A}=f \hat{s}$ is applied at $t<0$ [cf. Eq. (35)]. The perturbation is switched off at $t>0$, and the double well reverts to its unperturbed symmetric form. This, in turn, causes a relaxation, or regression, of the reactant and product populations to their equilibrium proportions with respect to the unperturbed potential. Upon application of linear response theory, an alternative expression to the reaction rate constant is obtained, namely Eq. (45). When visualized in this fashion, the choice of a perturbation linear in the reaction coordinate in some ways seems more natural than the choice $\hat{A}=h(\hat{s})$ made in the more conventional treatment [cf. Eqs. (39)-(43)].

The evaluation of the centroid symbols of the Heaviside and flux operators is straightforward, although rather tedious. The final results are as follows: 


$$
\begin{aligned}
h_{c}\left(s_{c}, p_{c}, \mathbf{Q}_{\mathbf{c}}, \mathbf{P}_{\mathbf{c}}\right) & \equiv \operatorname{Tr}\left[\hat{\delta}_{c}\left(s_{c}, p_{c}, \mathbf{Q}_{\mathbf{c}}, \mathbf{P}_{\mathbf{c}}\right) \hat{h}\right] \\
& =\frac{\rho_{c}^{+}\left(s_{c}, \mathbf{Q}_{\mathbf{c}}\right)}{\rho_{c}\left(s_{c}, \mathbf{Q}_{\mathbf{c}}\right)}, \\
F_{c}\left(s_{c}, p_{c}, \mathbf{Q}_{\mathbf{c}}, \mathbf{P}_{\mathbf{c}}\right) & \equiv \operatorname{Tr}\left[\hat{\delta}_{c}\left(s_{c}, p_{c}, \mathbf{Q}_{\mathbf{c}}, \mathbf{P}_{\mathbf{c}}\right) \hat{F}\right] \\
& =\frac{p_{c}}{m} \frac{\rho_{c}^{\prime}\left(s_{c}, \mathbf{Q}_{\mathbf{c}}\right)}{\rho_{c}\left(s_{c}, \mathbf{Q}_{\mathbf{c}}\right)},
\end{aligned}
$$

where

$$
\begin{aligned}
\rho_{c}\left(s_{c}, \mathbf{Q}_{\mathbf{c}}\right)= & \mathcal{C} \int_{s(0)=s(\beta \hbar)} D s(\tau) \int_{\mathbf{Q}(0)=\mathbf{Q}(\beta \hbar)} D \mathbf{Q}(\tau) \\
& \times \delta\left[s_{c}-(\beta \hbar)^{-1} \int_{0}^{\beta \hbar} d \tau s(\tau)\right] \\
& \times \delta\left[\mathbf{Q}_{\mathbf{c}}-(\beta \hbar)^{-1} \int_{0}^{\beta \hbar} d \tau \mathbf{Q}(\tau)\right] \\
& \times \exp \{-\mathcal{S}[s(\tau), \mathbf{Q}(\tau)] / \hbar\} \\
= & \lim _{P \rightarrow \infty} \mathcal{N}(P) \int d s_{1} \cdots \int d s_{P} \int d \mathbf{Q}_{1} \cdots \int d \mathbf{Q}_{\mathbf{P}} \\
& \times \delta\left[s_{c}-\frac{1}{P} \sum_{k=1}^{P} s_{k}\right] \delta\left[\mathbf{Q}_{\mathbf{c}}-\frac{1}{P} \sum_{k=1}^{P} \mathbf{Q}_{\mathbf{k}}\right] \\
& \times \exp \left\{-\mathcal{S}\left(s_{1}, \ldots, s_{P}, \mathbf{Q}_{\mathbf{1}}, \ldots, \mathbf{Q}_{\mathbf{P}}\right) / \hbar\right\},
\end{aligned}
$$$$
\rho_{c}^{+}\left(s_{c}, \mathbf{Q}_{\mathbf{c}}\right)=\mathcal{C} \int_{s(0)=s(\beta \hbar)} D s(\tau) \int_{\mathbf{Q}(0)=\mathbf{Q}(\beta \hbar)} D \mathbf{Q}(\tau)
$$$$
\times\left[(\beta \hbar)^{-1} \int_{0}^{\beta \hbar} d \tau h(s(\tau))\right]
$$$$
\times \delta\left[s_{c}-(\beta \hbar)^{-1} \int_{0}^{\beta \hbar} d \tau s(\tau)\right]
$$$$
\times \delta\left[\mathbf{Q}_{\mathbf{c}}-(\beta \hbar)^{-1} \int_{0}^{\beta \hbar} d \tau \mathbf{Q}(\tau)\right]
$$$$
\times \exp \{-\mathcal{S}[s(\tau), \mathbf{Q}(\tau)] / \hbar\}
$$$$
=\lim _{P \rightarrow \infty} \mathcal{N}(P) \int d s_{1} \cdots \int d s_{P} \int d \mathbf{Q}_{1} \cdots \int d \mathbf{Q}_{\mathbf{P}}
$$$$
\times\left[\frac{1}{P} \sum_{k=1}^{P} h\left(s_{k}\right)\right] \delta\left[s_{c}-\frac{1}{P} \sum_{k=1}^{P} s_{k}\right]
$$$$
\times \delta\left[\mathbf{Q}_{\mathbf{c}}-\frac{1}{P} \sum_{k=1}^{P} \mathbf{Q}_{\mathbf{k}}\right]
$$$$
\times \exp \left\{-\mathcal{S}\left(s_{1}, \ldots, s_{P}, \mathbf{Q}_{1}, \ldots, \mathbf{Q}_{\mathbf{P}}\right) / \hbar\right\} \text {, }
$$

$$
\begin{gathered}
+\sum_{i=1}^{N} \frac{1}{2} M^{(i)}\left[\dot{Q}^{(i)}(\tau)\right]^{2}+V_{0}(s(\tau)) \\
+V(\mathbf{Q}(\tau))+W(s(\tau), \mathbf{Q}(\tau))\}, \\
\frac{1}{\hbar} \mathcal{S}\left[s_{1}, \ldots, s_{P}, \mathbf{Q}_{1}, \ldots, \mathbf{Q}_{\mathbf{P}}\right]= \\
\quad \beta \sum_{k=1}^{P}\left\{\frac{1}{2} m \omega_{P}^{2}\left(s_{k}-s_{k+1}\right)^{2}\right. \\
+\sum_{i=1}^{N} \frac{1}{2} M^{(i)} \omega_{P}^{2}\left(Q_{k}^{(i)}-Q_{k+1}^{(i)}\right)^{2} \\
\quad+\frac{1}{P}\left[V_{0}\left(s_{k}\right)+V\left(\mathbf{Q}_{k}\right)\right. \\
\left.\left.\quad+W\left(s_{k}, \mathbf{Q}_{k}\right)\right]\right\} \\
\mathcal{C}=\left\{\left(\frac{2 \pi \beta \hbar^{2}}{m}\right) \prod_{i=1}^{N}\left(\frac{2 \pi \beta \hbar^{2}}{M^{(i)}}\right)\right\}^{1 / 2}, \\
\mathcal{N}(P)=\mathcal{C}\left\{\left(\frac{m P}{2 \pi \beta \hbar^{2}}\right) \prod_{i=1}^{N}\left(\frac{M^{(i)} P}{2 \pi \beta \hbar^{2}}\right)\right\}
\end{gathered}
$$

The discrete path-integral versions of the functions $\rho_{c}^{+}\left(s_{c}, \mathbf{Q}_{\mathbf{c}}\right)$ and $\rho_{c}^{\prime}\left(s_{c}, \mathbf{Q}_{\mathbf{c}}\right)$ are equivalent to configurational partition functions of a system consisting of $N+1$ chains, 
whose centroids are fixed at $\left(s_{c}, Q_{c}^{(1)}, \ldots, Q_{c}^{(N)}\right)$. In the case of $\rho_{c}^{+}\left(s_{c}, \mathbf{Q}_{\mathbf{c}}\right)$, the statistical weight for a given configuration is proportional to $\sum_{k=1}^{P} h\left(s_{k}\right) / P$, i.e., the fraction of the beads in the $s$ chain that lie to the right of the barrier top $(s>0)$. In the case of $\rho_{c}^{\prime}\left(s_{c}, \mathbf{Q}_{\mathbf{c}}\right)$, the statistical weight for a given configuration is proportional to $\Sigma_{k=1}^{P} \delta\left(s_{k}\right) / P$, i.e., the fraction of the beads in the $s$ chain that lie at the barrier top ( $s$ $=0)$. Alternatively, $\rho_{c}^{\prime}\left(s_{c}, \mathbf{Q}_{\mathbf{c}}\right)$ can be associated with the partition function of $N+1$ chains with fixed centroids and with one of the beads in the $s$ chain attached to the barrier top (the symmetry of the chain renders the actual identity of the bead attached to the barrier top unimportant).

It should be emphasized that Eq. (46) gives the exact quantum reaction rate constant. The CMD approximation is next introduced in order to treat the time evolution of the flux

$$
F_{c}\left(t ; s_{c}, p_{c}, \mathbf{Q}_{\mathbf{c}}\right) \approx F_{c}\left[s_{c}(t), p_{c}(t), \mathbf{Q}_{\mathbf{c}}(t)\right]
$$

[note that $F_{c}$, Eq. (48), is independent of $\mathbf{P}_{\mathbf{c}}$ ]. The centroid position-flux correlation function can now be approximated in the following way:

$$
\begin{aligned}
(2 \pi \hbar)^{N+1} Z C_{\hat{s}, \hat{F}}^{\text {Kubo }}(t) & =\int d s_{c} \int d p_{c} \int d \mathbf{Q}_{\mathbf{c}} \int d \mathbf{P}_{\mathbf{c}} \exp \left\{-\beta\left[\sum_{i=1}^{N} \frac{\left(P_{c}^{(i)}\right)^{2}}{2 M^{(i)}}+\frac{p_{c}^{2}}{2 m}+V_{c m}\left(s_{c}, \mathbf{Q}_{\mathbf{c}}\right)\right]\right\} s_{c} F_{c}\left(t ; s_{c}, p_{c}, \mathbf{Q}_{\mathbf{c}}\right) \\
& \approx \int d s_{c} \int d p_{c} \int d \mathbf{Q}_{\mathbf{c}} \int d \mathbf{P}_{\mathbf{c}} \exp \left\{-\beta\left[\sum_{i=1}^{N} \frac{\left(P_{c}^{(i)}\right)^{2}}{2 M^{(i)}}+\frac{p_{c}^{2}}{2 m}+V_{c m}\left(s_{c}, \mathbf{Q}_{\mathbf{c}}\right)\right]\right\} s_{c} F_{c}\left(s_{c}(t), p_{c}(t), \mathbf{Q}_{\mathbf{c}}(t)\right) \\
& =\int d s_{c} \int d p_{c} \int d \mathbf{Q}_{\mathbf{c}} \int d \mathbf{P}_{\mathbf{c}} \exp \left\{-\beta\left[\sum_{i=1}^{N} \frac{\left(P_{c}^{(i)}\right)^{2}}{2 M^{(i)}}+\frac{p_{c}^{2}}{2 m}+V_{c m}\left(s_{c}, \mathbf{Q}_{\mathbf{c}}\right)\right]\right\} s_{c}(-t) F_{c}\left(s_{c}, p_{c}, \mathbf{Q}_{\mathbf{c}}\right),
\end{aligned}
$$

where the second equality is based on the approximation in Eq. (55), and the third equality is based on the time reversibility of CMD correlation functions. Substituting Eq. (48) in the last equality of Eq. (56), and utilizing Eq. (10), leads to our final result

$$
(2 \pi \hbar)^{N+1} Z C_{\hat{s}, \hat{F}}^{\text {Kubo }}(t) \approx \int d s_{c} \int d p_{c} \int d \mathbf{Q}_{\mathbf{c}} \int d \mathbf{P}_{\mathbf{c}} \rho_{c}^{\prime}\left(s_{c}, \mathbf{Q}_{\mathbf{c}}\right) \exp \left\{-\beta\left[\sum_{i=1}^{N} \frac{\left(P_{c}^{(i)}\right)^{2}}{2 M^{(i)}}+\frac{p_{c}^{2}}{2 m}\right]\right\} s_{c}(-t) \frac{p_{c}}{m} .
$$

Thus, the task of calculating $C_{\hat{s}, \hat{F}}^{\text {Kubo }}(t)$ is transformed into the evaluation of a classical-like correlation between the reaction coordinate centroid momentum, $p_{c}$, at the initial time, and the backwards-in-time propagated reaction coordinate centroid position, $s_{c}(-t)$, with the initial (unnormalized) distribution of centroid positions given by $\rho_{c}^{\prime}\left(s_{c}, \mathbf{Q}_{\mathbf{c}}\right)$ [cf. Eq. (51)]. Placing the flux at the initial time is crucial for overcoming rare event statistics. In the classical limit, $F_{c}$ reduces to $p \delta(s) / m$ and hence $\rho_{c}^{\prime}\left(s_{c}, \mathbf{Q}_{\mathbf{c}}\right) / \rho_{c}\left(s_{c}, \mathbf{Q}_{\mathbf{c}}\right) \rightarrow \delta\left(s_{c}\right)$, such that only trajectories that start at $s_{c}=0$ are sampled. Quantum delocalization manifests itself by broadening this delta function, and the degree of broadening will depend on the typical delocalization of the $s$ chain when one of its beads is attached at the barrier top position.

The above discussion leads to the following approximate expression for the reaction rate constant:

$$
k \approx-\frac{\int d s_{c} \int d p_{c} \int d \mathbf{Q}_{\mathbf{c}} \int d \mathbf{P}_{\mathbf{c}} \rho_{c}^{\prime}\left(s_{c}, \mathbf{Q}_{\mathbf{c}}\right)^{\prime} \exp \left\{-\beta\left[\Sigma_{i=1}^{N} \frac{\left(P_{c}^{(i)}\right)^{2}}{2 M^{(i)}}+\frac{p_{c}^{2}}{2 m}\right]\right\} s_{c}\left(-t_{p}\right) p_{c} / m}{\int d s_{c} \int d p_{c} \int d \mathbf{Q}_{\mathbf{c}} \int d \mathbf{P}_{\mathbf{c}} \rho_{c}\left(s_{c}, \mathbf{Q}_{\mathbf{c}}\right) \exp \left\{-\beta\left[\Sigma_{i=1}^{N} \frac{\left(P_{c}^{(i)}\right)^{2}}{2 M^{(i)}}+\frac{p_{c}^{2}}{2 m}\right]\right\} \delta s_{c} \delta h_{c}\left(s_{c}, \mathbf{Q}_{\mathbf{c}}\right)} .
$$

The denominator in Eq. (58) can be calculated via equilibrium PIMD or PIMC simulations. The correlation function in the numerator of Eq. (58) can be calculated in two steps: (1) sampling of the initial centroid positions, $\left\{s_{c}(0), \mathbf{Q}_{\mathbf{c}}(0)\right\}$, and momenta, $\left\{p_{c}(0), \mathbf{P}_{\mathbf{c}}(0)\right\}$, via a PIMD or PIMC simulation, ${ }^{11,12}$ with the constraint $s_{1}=0$ (the cyclic symmetry renders the actual label of the constrained bead physically meaningless); (2) propagating the sampled centroid positions and momenta via a constraint-free CMD simulation. The correlation function can then be obtained by averaging over a large number of trajectories that start at different, randomly sampled, initial states.

It is interesting to contrast the above CMD-based reac- tion rate constant, Eq. (58), to the PI-QTST reaction rate constant. $^{2-6}$ The most general form of the latter is given by

$$
P_{R}^{e q} P_{P}^{e q} k_{\mathrm{PI}-\mathrm{QTST}}=\frac{\kappa_{\mathrm{opt}}}{2 \pi \beta \hbar} \frac{\int d \mathbf{Q}_{\mathbf{c}} \int d s_{c} \delta\left(s_{c}\right) \rho_{c}\left(s_{c}, \mathbf{Q}_{\mathbf{c}}\right)}{\int d \mathbf{Q}_{\mathbf{c}} \int d s_{c} \boldsymbol{\rho}_{c}\left(s_{c}, \mathbf{Q}_{\mathbf{c}}\right)},
$$

where $\kappa_{\text {opt }}$ is the classical Grote-Hynes transmission coefficient at the variationally optimized barrier frequency. Like Eq. (58), it involves constrained sampling. However, the actual constraints are different: $s_{1}=0$ in the case of Eq. (58) and $s_{c}=0$ in the case of Eq. (59). It should also be noted that Eq. (58) was derived directly from the CMD formalism, and is therefore expected to include dynamical effects, such as 
TABLE I. The parameters for the DW1 model. $E_{b}=a_{1}^{2} / 4 a_{2}$ is the barrier height; $\omega_{b}=\sqrt{2 a_{1} / m}$ is the barrier frequency; $\omega_{0}=\sqrt{2} \omega_{b}$ is the well frequency. The parameters are given in units of $\mathrm{cm}^{-1}$.

\begin{tabular}{lccc}
\hline \hline$E_{b}$ & $\omega_{b}$ & $\omega_{0}$ & $\omega_{c}$ \\
\hline 2085 & 500 & 707 & 500 \\
\hline \hline
\end{tabular}

the Kramers turnover, which PI-QTST cannot account for. The relationship between PI-QTST and CMD was the subject of a recent paper by Jang and Voth. ${ }^{6}$ These authors found that deriving PI-QTST from CMD requires additional approximations beyond these pertinent to CMD and TST. ${ }^{6}$ Hence, despite the fact that both of these approximate methods are based on the centroid concept, one should not expect them to give the exact same results.

\section{APPLICATION TO A DOUBLE WELL BILINEARLY COUPLED TO A HARMONIC BATH}

In this section, the new method described above is used to calculate reaction rate constants in the case of a symmetric double-well potential bilinearly coupled to a harmonic bath. It should be emphasized that the method is by no means limited to such systems, and can easily accommodate anharmonic environments, nonlinear coupling, and multidimensional reactive potential surfaces. However, the availability of numerically exact quantum rate constants for a wide range of temperatures and frictions in the case of the harmonic bath ${ }^{17}$ provides an opportunity for testing the accuracy of the new method.

\section{A. The model}

Following Topaler and Makri, ${ }^{17}$ we consider a double well bilinearly coupled to a bath of harmonic oscillators. The total Hamiltonian operator is given by

$$
\begin{aligned}
\hat{H}= & \frac{\hat{p}^{2}}{2 m}+V_{0}(\hat{s})+\sum_{j}\left[\frac{\left(\hat{P}^{(j)}\right)^{2}}{2 M^{(j)}}+\frac{1}{2} M^{(j)}\left(\omega^{(j)}\right)^{2}\right. \\
& \left.\times\left(\hat{Q}^{(j)}-\frac{c^{(j)} \hat{s}}{M^{(j)}\left(\omega^{(j)}\right)^{2}}\right)^{2}\right] .
\end{aligned}
$$

The potential along the bare reaction coordinate, $V_{0}(s)$, is taken to be of the form of a symmetric double well,

$$
V_{0}(s)=-a_{1} s^{2}+a_{2} s^{4} \text {. }
$$

It should be noted that $V_{0}(s)$ is defined such that the barrier top is positioned at $s=0$, as required. The spectral density of the bath is assumed to be ohmic, with an exponential cutoff

$$
J(\omega)=\frac{\pi}{2} \sum_{j} \frac{\left(c^{(j)}\right)^{2}}{M^{(j)} \omega^{(j)}} \delta\left(\omega-\omega^{(j)}\right)=\eta \omega e^{-\omega / \omega_{c}},
$$

where $\eta$ is the friction coefficient (in the limit $\omega_{c} \rightarrow \infty$ ). $m$ was taken to be the mass of a proton, and the values of the parameters defining $V_{0}(s)$ and $J(\omega)$ were taken to be the same as in the DW1 model of Makri and Topaler ${ }^{17}$ (cf. Table I).

\section{B. Discretization of the bath}

In order to perform the simulations, the continuous bath spectral density of Eq. (62) had to be discretized. ${ }^{17,26,66}$ To this end, it is convenient to work with the mass-weighted coordinates and momenta of the bath modes, $\left\{\widetilde{Q}^{(j)}\right.$ $\left.=\sqrt{M^{(j)}} \hat{Q}^{(j)}\right\}$ and $\left\{\widetilde{P}^{(j)}=\hat{P}^{(j)} / \sqrt{M^{(j)}}\right\}$, respectively, such that

$$
\begin{aligned}
\hat{H}= & \frac{\hat{p}^{2}}{2 m_{0}}+V_{0}(\hat{s})+\sum_{j}\left[\frac{\widetilde{P}_{j}^{2}}{2}+\frac{1}{2}\left(\omega^{(j)}\right)^{2}\right. \\
& \left.\times\left(\widetilde{Q}_{j}-\frac{c^{(j)} \hat{s}}{\sqrt{M^{(j)}}\left(\omega^{(j)}\right)^{2}}\right)^{2}\right] .
\end{aligned}
$$

This form makes it clear that each bath mode is characterized by two, rather than three, parameters: $\omega^{(j)}$ and $c^{(j)} / \sqrt{M^{(j)}}$. Next, a density number of bath modes, $\rho(\omega)$, is chosen. In the actual simulations presented below, $\rho(\omega) \propto J(\omega) / \omega$. The frequencies of the discrete bath modes, $\left\{\omega^{(j)}\right\}$, are obtained as follows:

$$
\int_{0}^{\omega^{(j)}} d \omega \rho(\omega)=j, \quad j=1,2, \ldots, N,
$$

with $N$ large enough such that $\omega_{N} \gg \omega_{c}$. The values of $\left\{c^{(j)} / \sqrt{M^{(j)}}\right\}$ are then obtained from ${ }^{17}$

$$
\frac{\left(c^{(j)}\right)^{2}}{M^{(j)}}=\frac{2}{\pi} \omega^{(j)} \frac{J\left(\omega^{(j)}\right)}{\rho\left(\omega^{(j)}\right)} .
$$

For the simulations reported here, convergence was achieved at $N=100-150$.

\section{Classical dynamics simulations}

The new methodology for calculating reaction rate constants was first tested in the classical limit. The results are presented in terms of the transmission coefficient

$$
\kappa=k_{\mathrm{PR}} / k_{\mathrm{PR}}^{\mathrm{TST}},
$$

where

$$
k_{\mathrm{PR}}^{\mathrm{TST}}=\frac{1}{m} \frac{\langle\delta(s) p h(p)\rangle}{\langle 1-h(s)\rangle}
$$

is the classical TST reactant-to-product reaction rate constant $(\langle\ldots\rangle$ corresponds to averaging over the classical many-body Boltzmann distribution). The classical reactant-to-product reaction rate constant in the case of a symmetrical double well is given by

$$
k_{\mathrm{PR}}^{\mathrm{Cl}}=\frac{k^{\mathrm{Cl}}}{2}=-\frac{1}{2 m} \frac{\langle A(-t) p \delta(s)\rangle}{\langle\delta h \delta A\rangle} .
$$

In Eq. (68), $A$ can be any perturbation that distorts the equilibrium reactant and product populations. Substituting Eqs. (67) and (68) into Eq. (66), and using the fact that the double-well potential is symmetric, we obtain

$$
\kappa^{\mathrm{Cl}}=\frac{k_{\mathrm{PR}}^{\mathrm{Cl}}}{k_{\mathrm{PR}}^{\mathrm{TST}}}=-\sqrt{\frac{\pi \beta}{8 m}} \frac{1}{\langle\delta A \delta h\rangle} \frac{\langle A(-t) p \delta(s)\rangle}{\langle\delta(s)\rangle} .
$$



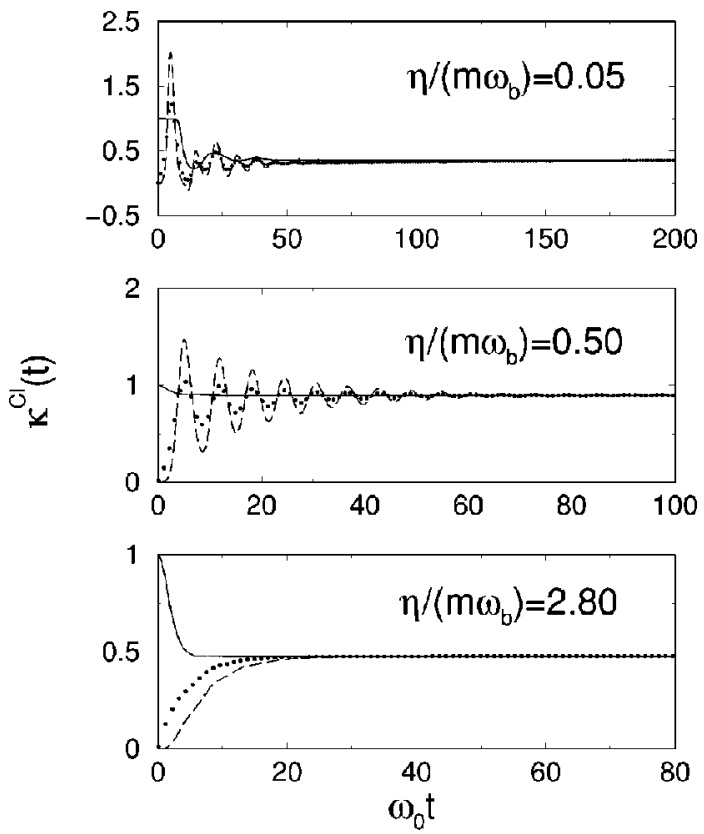

FIG. 2. The approach of the classical transmission coefficient to its asymptotic value. The plots were obtained for DW1 at $200 \mathrm{~K}$. The upper panel corresponds to low friction (below the Kramers crossover), the middle panel corresponds to intermediate friction (around the Kramers crossover), and the lower panel corresponds to high friction (above the Kramers crossover). The different lines correspond to different choices of the perturbation, $A: A=h(s)$ (solid line), $A=s$ (circles), $A=s^{3}$ (dashed line).

The equilibrium average $\langle\delta A \delta h\rangle$ was calculated via NoséHoover chain classical MD simulations, using the VV-3 algorithm of Jang and Voth ${ }^{67}$ (Nosé-Hoover chains of length four were attached to each of the degrees of freedom).

The term $\langle A(-t) p \delta(s)\rangle /\langle\delta(s)\rangle$ was evaluated from a separate simulation, by averaging over many trajectories that started at $s=0$. Since the bath modes are uncoupled at $s$ $=0$, their initial states could be randomly sampled from the phase-space Gaussian Boltzmann distributions of the individual harmonic oscillators. The sampled initial states were then propagated backwards in time by the velocity-Verlet algorithm (without Nosé-Hoover thermostats).

The approach of the classical time-dependent transmission coefficient to its asymptotic value is shown in Fig. 2 for different choices of the perturbation, $A$. As can be clearly seen, different choices of $A-A=h(s)$ (solid line), $A=s$ (circles), and $A=s^{3}$ (dashed line)—lead to exactly the same asymptotic value, and hence the same reaction rate constant. Different choices of $A$ may differ, however, in how long it takes to reach the plateau. The choice of $A=h(s)$ is the quickest due to its insensitivity to oscillations within the reactant and product wells. The signature of these oscillations is clearly seen in the low friction behavior or $\kappa^{\mathrm{Cl}}(t)$ when $A=s$ and $s^{3}$. Increasing the friction leads to damping of these oscillations, and the time it takes to reach the plateau becomes independent of the choice of $A$. We were able to reproduce the classical rate constants and demonstrate the equivalence of different choices of $A$ across the rather wide range of temperatures and frictions considered by Makri and Topaler. ${ }^{17}$

\section{CMD simulation techniques}

Performing a CMD simulation of a system consisting of $N+1$ degrees of freedom would generally involve PIMD or PIMC simulations involving $N+1$ coupled chains, each consisting of $P$ beads. However, in our case, we can take advantage of the fact that harmonic degrees of freedom can be averaged analytically. In particular, when evaluating the centroid distribution, $\rho_{c}\left(s_{c}, \mathbf{Q}_{\mathbf{c}}\right)$, one can analytically average over the $N$ harmonic bath modes. This leads to the following result: ${ }^{68}$

$$
\begin{aligned}
\rho_{c}\left(s_{c}, \mathbf{Q}_{\mathbf{c}}\right)= & \mathcal{A}(P) \\
& \times \exp \left\{-\beta\left[V_{\mathrm{eff}}\left(s_{c}\right)+\sum_{j=1}^{N} \frac{1}{2} M^{(j)}\left(\omega^{(j)}\right)^{2}\right.\right. \\
& \left.\left.\times\left(Q_{c}^{(j)}-\frac{c^{(j)} s_{c}}{M^{(j)}\left(\omega^{(j)}\right)^{2}}\right)^{2}\right]\right\}
\end{aligned}
$$

where

$$
\begin{aligned}
\exp [- & \left.\beta V_{\mathrm{eff}}\left(s_{c}\right)\right] \\
= & \int d \widetilde{s}_{1} \cdots \int d \widetilde{s}_{P} \delta\left(\widetilde{s}_{1}-s_{c}\right) \\
& \quad \times \exp \left\{-\beta\left[\sum_{k=2}^{P} \frac{1}{2}\left[m \Omega_{k}^{2}+\Omega_{k} \hat{\eta}\left(\Omega_{k}\right)\right] \widetilde{s}_{k}^{2}\right.\right. \\
& \left.\left.+\frac{1}{P} \sum_{k=1}^{P} V\left[s_{k}\left(\widetilde{s}_{1}, \ldots, \widetilde{s}_{P}\right)\right]\right]\right\},
\end{aligned}
$$

and

$\mathcal{A}(P)$

$$
\begin{aligned}
= & \sqrt{\frac{2 \pi \hbar^{2} \beta}{m}}\left(\frac{m P}{2 \pi \beta \hbar^{2}}\right)^{P / 2} \prod_{i=1}^{N}\left[\sqrt{\frac{2 \pi \hbar^{2} \beta}{M^{(i)}}}\left(\frac{M^{(i)} P}{2 \pi \beta \hbar^{2}}\right)^{P / 2}\right] \\
& \times \prod_{i=1}^{N} \prod_{k=2}^{P} \sqrt{\frac{2 \pi}{\beta M^{(i)}\left(\Omega_{k}^{2}+\omega_{i}^{2}\right)}} .
\end{aligned}
$$

Here, $\left\{\widetilde{s}_{k}\right\}$ and $\left\{\Omega_{k}\right\}$ are the coordinates and frequencies of the normal modes of the $s$ chain

$$
\left.\begin{array}{l}
\widetilde{s}_{k}=\frac{1}{P} \sum_{l=1}^{P} U_{l k} s_{l}, \quad U_{l 1}=1, \quad U_{l P}=-(-1)^{l}, \\
\Omega_{1}=0, \quad \Omega_{P}=\frac{2 P}{\beta \hbar}, \\
U_{k, 2 n-2}=\sqrt{2} \cos [2 \pi(k-1)(n-1) / P] \\
U_{k, 2 n-1}=-\sqrt{2} \sin [2 \pi(k-1)(n-1) / P] \\
\Omega_{2 n-1}=\Omega_{2 n-2}=\frac{P}{\beta \hbar} \sqrt{2[1-\cos (2 \pi(n-1) / P)]} \\
\quad(n=2,3, \ldots, P / 2),
\end{array}\right\}
$$

and $\hat{\eta}(z)$ is the Laplace transform of the classical dynamical friction kernel 


$$
\hat{\eta}(z)=\sum_{i=1}^{N} \frac{\left(c^{(i)}\right)^{2}}{M^{(i)}\left(\omega^{(i)}\right)^{2}} \frac{z}{z^{2}+\left(\omega^{(i)}\right)^{2}} .
$$

Equations (70) and (71) reveal that our system is equivalent to that consisting of a classical chain whose beads are positioned at $\left\{s_{1}, \ldots, s_{P}\right\}$, coupled to $N$ classical-like bath modes whose coordinates are given by $Q_{c}^{(1)}, \ldots, Q_{c}^{(N)}$. The $\hat{\eta}$-dependent terms correspond to bath-induced shifts to the frequencies of the springs that connect the beads. Increasing the friction makes these springs stiffer, thereby diminishing quantum delocalization. The second way in which the bath affects the $s$ chain is via the coupling term between the centroids of the bath modes, $\left\{Q_{c}^{(i)}\right\}$, and the centroid of the $s$ chain, $s_{c}$. The corresponding interaction potential takes almost the exact same form as the classical interaction potential, except for the fact that the classical positions are replaced by the corresponding centroid positions. Similar expressions can be obtained for $\rho_{c}^{\prime}\left(s_{c}, \mathbf{Q}_{\mathbf{c}}\right)$ and $\rho_{c}^{+}\left(s_{c}, \mathbf{Q}_{\mathbf{c}}\right)$ by adding the corresponding constraints to Eq. (71).

The calculation of the reaction rate constant from CMD, Eq. (58), was carried out via two separate simulations. The first simulation was designed for calculating the denominator, which in this case can be put in the following form:

$$
\begin{aligned}
& \int d s_{c} \int d p_{c} \int d \mathbf{Q}_{\mathbf{c}} \int d \mathbf{P}_{\mathbf{c}} \rho_{c}\left(s_{c}, p_{c}, \mathbf{Q}_{\mathbf{c}}, \mathbf{P}_{\mathbf{c}}\right) \delta h_{c}\left(s_{c}, \mathbf{Q}_{\mathbf{c}}\right) \delta s_{c} \\
& \int d s_{c} \int d p_{c} \int d \mathbf{Q}_{\mathbf{c}} \int d \mathbf{P}_{\mathbf{c}} \rho_{c}\left(s_{c}, p_{c}, \mathbf{Q}_{\mathbf{c}}, \mathbf{P}_{\mathbf{c}}\right) \\
& =\frac{1}{P} \frac{\int d \widetilde{s}_{1} \cdots \int d \widetilde{s}_{P} e^{-\beta\left[\Sigma_{k=2}^{P} 1 / 2\left[m \Omega_{k}^{2}+\Omega_{k} \hat{\eta}\left(\Omega_{k}\right)\right] \widetilde{s}_{k}^{2}+1 / P \Sigma_{k=1}^{P} V\left[s_{k}\left(\widetilde{s}_{1}, \ldots, \widetilde{s}_{P}\right)\right]\right]} \widetilde{s}^{(1)} \sum_{k=1}^{P} h\left[s_{k}\left(\widetilde{s}_{1}, \ldots, \widetilde{s}_{P}\right)\right]}{\int d \widetilde{s}_{1} \cdots \int d \widetilde{s}_{P} e^{-\beta\left[\Sigma_{k=2}^{P}\right.}}
\end{aligned}
$$

To this end, we performed non-Boltzmann sampling ${ }^{69}$ over the independent Gaussian distribution of the normal mode coordinates, $\left\{\widetilde{s}_{1}, \ldots, \widetilde{s}_{P}\right\}$, and averaged over the term that couples them. Since $\Omega_{1}=0, \widetilde{s}_{1}=s_{c}$ was sampled from $-L$ to $L$ with equal probability, and the value of $L$ was increased until we reached convergence.

The second simulation was designed for calculating the numerator in Eq. (58)

$$
\begin{aligned}
& \frac{\int d s_{c} \int d p_{c} \int d \mathbf{Q}_{\mathbf{c}} \int d \mathbf{P}_{\mathbf{c}} \rho_{c}\left(s_{c}, p_{c}, \mathbf{Q}_{\mathbf{c}}, \mathbf{P}_{\mathbf{c}}\right) \delta s_{c}(-t) F_{c}\left(s_{c}, p_{c}, \mathbf{Q}_{\mathbf{c}}\right)}{\int d s_{c} \int d p_{c} \int d \mathbf{Q}_{\mathbf{c}} \int d \mathbf{P}_{\mathbf{c}} \boldsymbol{\rho}_{c}\left(s_{c}, p_{c}, \mathbf{Q}_{\mathbf{c}}, \mathbf{P}_{\mathbf{c}}\right)} \\
& \quad=\left(\frac{\int d s_{c} \int d \mathbf{Q}_{\mathbf{c}} \boldsymbol{\rho}_{c}^{\prime}\left(s_{c}, \mathbf{Q}_{\mathbf{c}}\right)}{\int d s_{c} \int d \mathbf{Q}_{\mathbf{c}} \boldsymbol{\rho}_{c}\left(s_{c}, \mathbf{Q}_{\mathbf{c}}\right)}\right)\left(\frac{\int d s_{c} \int d p_{c} \int d \mathbf{Q}_{\mathbf{c}} \int d \mathbf{P}_{\mathbf{c}} \rho_{c}^{\prime}\left(s_{c}, p_{c}, \mathbf{Q}_{\mathbf{c}}, \mathbf{P}_{\mathbf{c}}\right) s_{c}(-t) p_{c} / m}{\int d s_{c} \int d p_{c} \int d \mathbf{Q}_{\mathbf{c}} \int d \mathbf{P}_{\mathbf{c}} \boldsymbol{\rho}_{c}^{\prime}\left(s_{c}, p_{c}, \mathbf{Q}_{\mathbf{c}}, \mathbf{P}_{\mathbf{c}}\right)}\right) .
\end{aligned}
$$

The first factor on the right-hand side was calculated via non-Boltzmann sampling over the Gaussian distribution of the independent normal modes, as described above, either with or without constraining one of the beads to $s=0$. The second factor required the appropriate initial sampling of the centroid of the constrained chain, $s_{c}$, which can be conveniently performed in the normal mode representation. The bath modes centroids, $\left\{Q_{c}^{(k)}\right\}$, for a given value of $s_{c}$, were sampled from the shifted Gaussian distribution, $e^{-\beta / 2 M^{(k)}\left(\omega^{(k)}\right)^{2}\left[Q_{c}^{(k)}-c^{(k)} s_{c} / M^{(k)}\left(\omega^{(k)}\right)^{2}\right]^{2}}$. Each sampled initial configuration was then propagated in time via a CMD simulation. The dependence of the centroid force on $s_{c}$ was tabulated beforehand, and the numerical effort involved in running a CMD simulation was therefore essentially the same as that of running a classical MD simulation.

The constrained centroid distribution, $\rho_{c}^{\prime}\left(s_{c}\right)$, deserves special attention. At high temperatures, this distribution is very narrow and localized at the barrier top. Its broadening as the temperature is lowered is a reflection of quantum delocalization. This behavior is demonstrated in Figs. 3-5. At the relatively high temperature of $300 \mathrm{~K}$ (cf. Fig. 3), the distribution is found to be unimodal and fairly localized

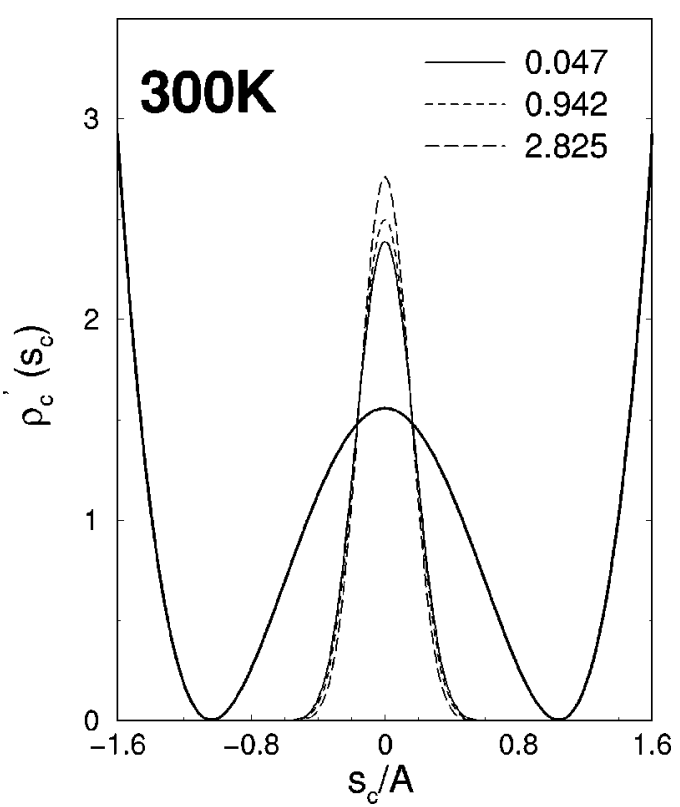

FIG. 3. The initial constrained centroid distribution $\rho_{c}^{\prime}\left(s_{c}\right)$ (normalized), for DW1 at $300 \mathrm{~K}$. The distribution is shown for various values of $\eta /\left(m \omega_{b}\right)$ (see the legends). The classical potential along the reaction coordinate is also shown for reference. 


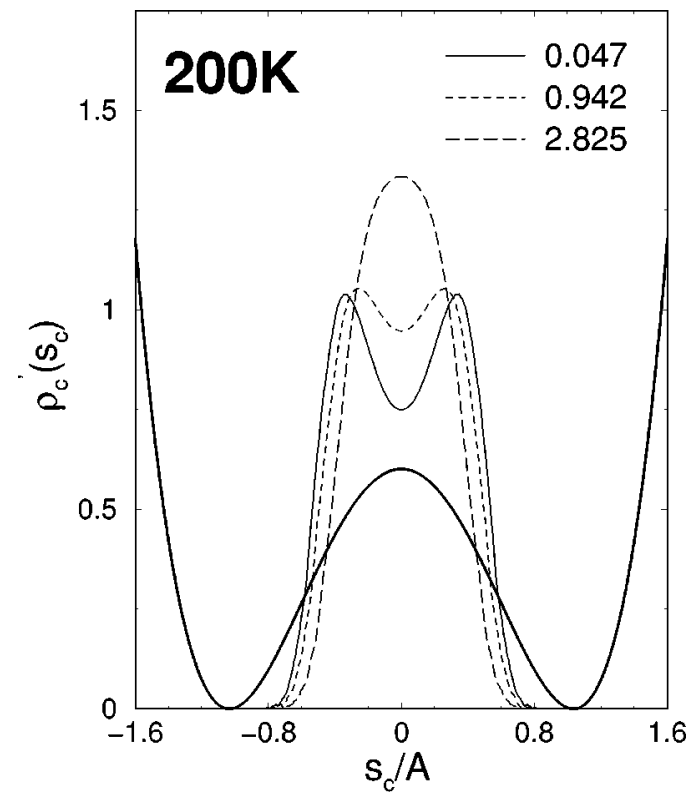

FIG. 4. Same as Fig. 2 except $T=200 \mathrm{~K}$.

around the barrier top. As expected, increasing the friction further localizes the distribution. A somewhat different picture emerges at $200 \mathrm{~K}$ (cf. Fig. 4). At high frictions, the distribution is wider than at $300 \mathrm{~K}$, but still unimodal and localized around the barrier top. However, the distribution becomes bimodal at low frictions. This is because lowering the temperature and/or friction leads to more extended chains. One of the beads has to be attached to the barrier top, but the rest of the beads seek regions of lower potential energy which are downhill on both sides of the barrier. As a result, the corresponding centroid distribution acquires a symmetric, with respect to the barrier top, bimodal structure. This behavior is further enhanced at $100 \mathrm{~K}$, where $\rho_{c}^{\prime}\left(s_{c}\right)$ is

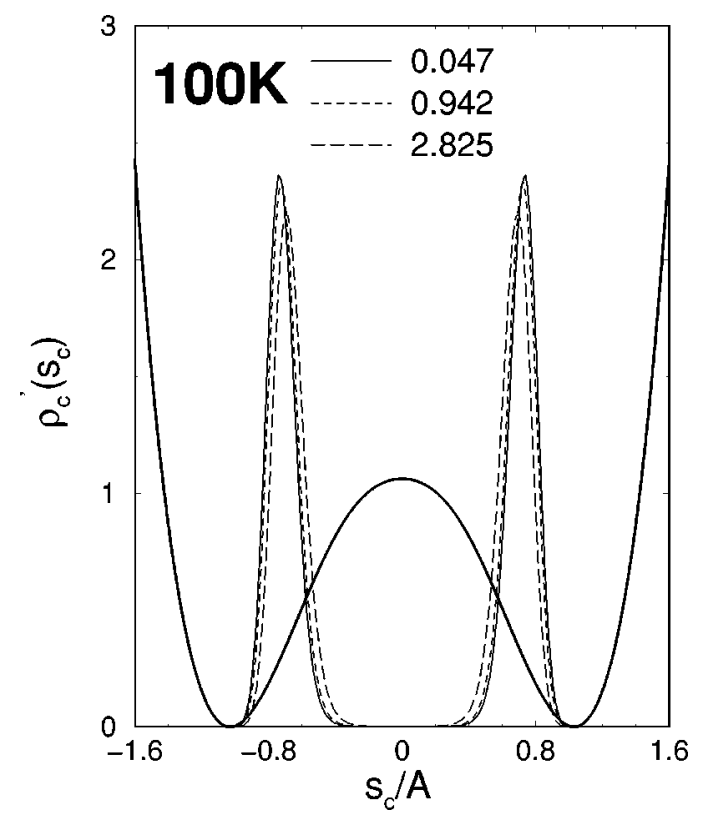

FIG. 5. Same as Fig. 2 except $T=100 \mathrm{~K}$.

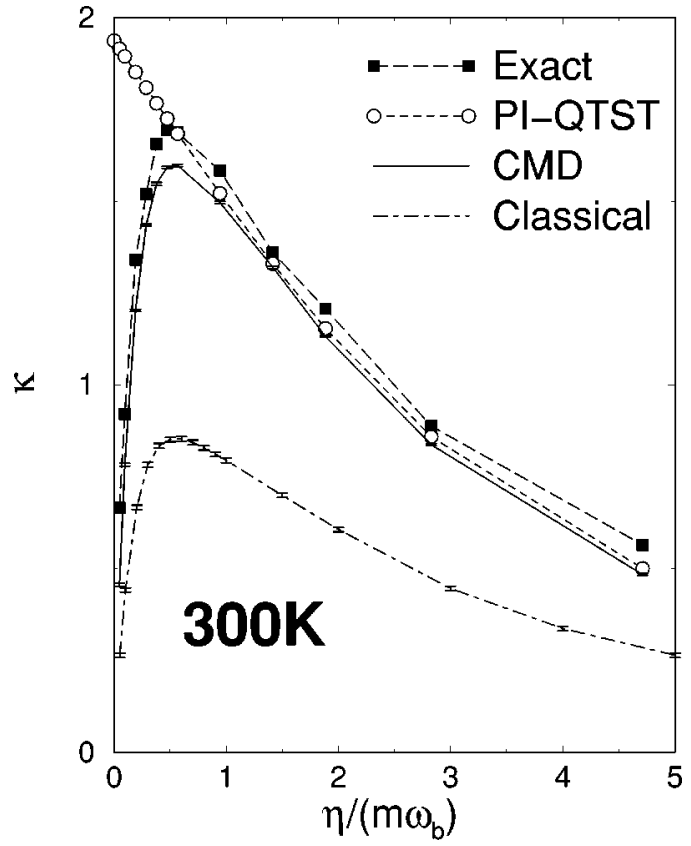

FIG. 6. The transmission coefficient as a function of friction, for DW1 at $300 \mathrm{~K}$. Shown are the exact, CMD, PI-QTST, and classical results.

seen to consist of two, clearly separated peaks on both sides of the barrier (cf. Fig. 5).

The above-mentioned behavior of $\rho_{c}^{\prime}\left(s_{c}\right)$ can lead to inefficient sampling at low temperatures, since the most likely starting centroid positions will have a low likelihood for crossing the barrier. In order to enhance the efficiency, we performed the sampling at $100 \mathrm{~K}$ with two infinitely high reflecting walls when $s_{c}$ is equal $-L$ or $L$. Starting with the walls in the close vicinity of the barrier top, $L$ is gradually increased until convergence is reached. The value of $L$ at which convergence is attained increases when the temperature is further decreased, due to quantum delocalization, thereby rendering even the above-mentioned sampling method inefficient below $100 \mathrm{~K}$. The development of methods to enhance the efficiency of the sampling at such very low temperatures will be the subject of future research.

\section{E. Results of CMD simulations}

Using the simulation techniques described above, we were able to calculate reaction rate constants for DW1 at $T$ $=100,200$, and $300 \mathrm{~K}$, for a wide range of frictions. The results of these calculations are presented in Figs. 6-8, alongside the numerically exact results, the results obtained from classical MD simulations, and the results from PIQTST. The following observations can be made based on these plots:

(1) The most important observation is that CMD captures most of the quantum enhancement to the reaction rate. Thus, the CMD approximation is seen to work well and lead to quantitative predictions in situations where classical mechanics fails.

(2) The CMD rate constant, Eq. (58), provides a lower bound to the exact results. This is a manifestation of the fact that CMD approximates the quantum dynamics, and there- 


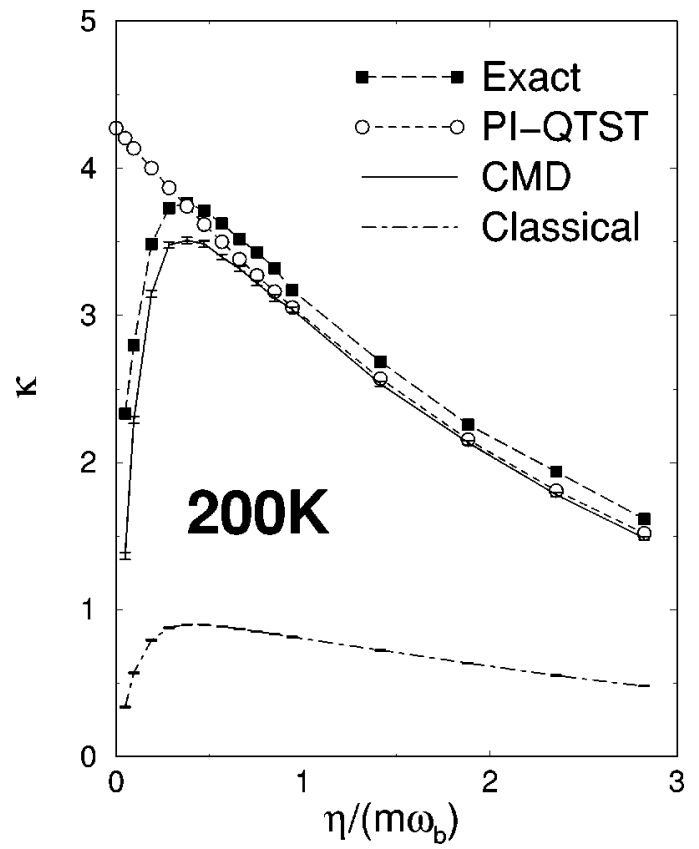

FIG. 7. The transmission coefficient as a function of friction, for DW1 at $200 \mathrm{~K}$. Shown are the exact, CMD, PI-QTST, and classical results.

fore misses some of the quantum enhancement. It should be emphasized, however, that the deviations from the exact results are usually rather small.

(3) CMD improves as the temperature increases. The relative weight of the quantum enhancement intensifies at lower temperatures, so that the relative error involved in approximating it becomes larger. However, it should be emphasized that CMD can still provide quantitative estimates at temperatures as low as $100 \mathrm{~K}$.

(4) CMD improves as the friction is increased. This is a manifestation of the fact that CMD cannot capture purely

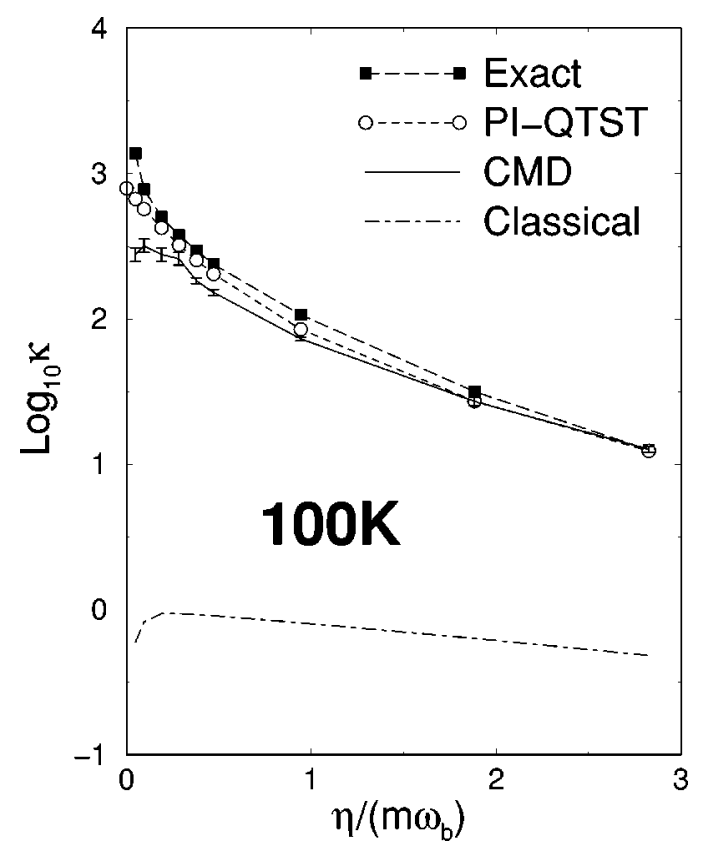

FIG. 8. The $\log _{10}$ of the transmission coefficient as a function of friction, for DW1 at $100 \mathrm{~K}$. Shown are the exact, CMD, PI-QTST, and classical results. quantum coherences for long times. These coherences are dephased by the bath at higher frictions, thereby improving the quality of CMD. This means that the present CMD-based method for computing rate constants will be applicable to many condensed phase systems for which the high friction region is the relevant one.

(5) The reaction rate constants obtained from CMD coincide with these obtained from PI-QTST at high frictions. At intermediate frictions, the predictions of PI-QTST are slightly better, which is likely to be accidental. PI-QTST, being a TST method, fails to capture the the turnover behavior at the low friction, energy diffusion regime. On the other hand, CMD, being a dynamical method, does capture the turnover behavior. These results indicate that CMD and PIQTST are distinctly different methods, despite the fact that they are both based on the centroid concept, with CMD a more general theoretical framework which includes dynamical effects.

\section{CONCLUSIONS}

A new method for calculating quantum reaction rate constants has been presented in this work. The new method is based on the following two elements:

(1) Linear-response theory, either classical or quantummechanical, leads to a family of expressions for the reaction rate constant. In all of these expressions, the rate constant is given in terms of a correlation function of the flux and a second observable. The important point is that the same value of the reaction rate constant will be obtained regardless of which second observable is chosen. In particular, one can express the quantum reaction rate constant in terms of a position-flux correlation function.

(2) CMD can be used to approximate the quantum position-flux correlation function, and hence the quantum reaction rate constant, in general many-body systems.

The method was tested on a model system consisting of a symmetric double well coupled to a harmonic bath. CMD was found to provide a very good approximation for the quantum reaction rate constant, and typically accounted for the major part of the quantum enhancement. It should be noted that the level of agreement with the numerically exact results is of similar quality as for rate constants calculations based on analytical continuation ${ }^{28,29}$ and semiclassical ${ }^{22,23,26}$ methods. Comparison to the linearized version of the semiclassical approximation ${ }^{23}$ is also worth noting. In this case, the semiclassical approximation amounts to doing the initial sampling based on the Wigner distribution of the combined Boltzmann and flux operators, followed by fully classical dynamics. CMD, on the other hand, is based on nonclassical sampling followed by classical-like dynamics on a nonclassical centroid potential. Despite the similar spirit of the two approaches, the additional important differences should also be highlighted: (1) the initial centroid distribution function is fundamentally different from the Wigner distribution, ${ }^{39}$ and (2) calculating the centroid distribution for realistic systems is feasible, while calculating the Wigner distribution for realistic systems may be extremely difficult due to its regions of negative amplitude. 
The sensitivity of the CMD approximation to the temperature and friction was systematically mapped, for the first time, within the context of a widely studied "many-body" system. It should be emphasized that the new method described herein is in no way limited to one-dimensional systems bilinearly coupled to harmonic baths. The application of the theory to more complex and realistic condensed phase systems will be the subject of future work.

Finally, it should be noted that the present method is able to accommodate alternative methods for computing the dynamics of centroid variables. ${ }^{40}$ Such methods may replace the CMD approximation in Eq. (55), and thus lead to potentially even more accurate methods for calculating quantum reaction rate constants.

\section{ACKNOWLEDGMENTS}

This work was supported by the National Science Foundation (No. CHE-9712884), and by start-up funding from the University of Michigan. One of the authors (E.G.) is grateful to Dr. Seogjoo Jang for enlightening correspondence regarding CMD and also thanks Professor David Tannor for introducing him to the problem of calculating quantum reaction rate constants, Dr. Udo Schmitt and Dr. Soonmin Jang for their help in writing the CMD code, and Professor Nancy Makri for sending an electronic copy of her data.

${ }^{1}$ P. Hänggi, P. Talkner, and M. Borkovec, Rev. Mod. Phys. 62, 251 (1990).

${ }^{2}$ G. A. Voth, D. Chandler, and W. H. Miller, J. Chem. Phys. 91, 7749 (1989).

${ }^{3}$ G. A. Voth, Chem. Phys. Lett. 170, 289 (1990).

${ }^{4}$ G. A. Voth, J. Phys. Chem. 97, 8365 (1993).

${ }^{5}$ G. A. Voth, Adv. Chem. Phys. 93, 135 (1996).

${ }^{6}$ S. Jang and G. A. Voth, J. Chem. Phys. 112, 8747 (2000).

${ }^{7}$ M. J. Gillan, Phys. Rev. Lett. 58, 563 (1987).

${ }^{8}$ M. J. Gillan, J. Phys. C 20, 3621 (1987).

${ }^{9}$ R. P. Feynman and A. R. Hibbs, Quantum Mechanics and Path Integrals (McGraw-Hill, New York, 1965).

${ }^{10}$ R. P. Feynman, Statistical Mechanics (Benjamin, New York, 1972).

${ }^{11}$ B. J. Berne and D. Thirumalai, Annu. Rev. Phys. Chem. 37, 401 (1986).

${ }^{12}$ D. M. Ceperley, Rev. Mod. Phys. 67, 279 (1995).

${ }^{13}$ P. Pechukas, Dynamics of Molecular Collisions, Part 2 (Plenum, New York, 1976), p. 269.

${ }^{14}$ T. Yamamoto, J. Chem. Phys. 33, 281 (1960).

${ }^{15}$ W. H. Miller, J. Chem. Phys. 61, 1823 (1974).

${ }^{16}$ W. H. Miller, S. D. Schwartz, and J. W. Tromp, J. Chem. Phys. 79, 4889 (1983).

${ }^{17}$ M. Topaler and N. Makri, J. Chem. Phys. 101, 7500 (1994).

${ }^{18}$ N. Makri and K. Thompson, Chem. Phys. Lett. 291, 101 (1998).

${ }^{19}$ R. P. Feynman and F. L. Vernon, Jr., Ann. Phys. 24, 118 (1963).

${ }^{20}$ U. Weiss, Quantum Dissipative Systems (World Scientific, London, 1993).

${ }^{21}$ N. Makri, J. Phys. Chem. A 102, 4414 (1998).

${ }^{22}$ W. H. Miller, Faraday Discuss. 110, 1 (1998).

${ }^{23}$ H. Wang, X. Sun, and W. H. Miller, J. Chem. Phys. 108, 9726 (1998).

${ }^{24}$ J. S. Shao and N. Makri, J. Phys. Chem. A 103, 7753 (1999).
${ }^{25}$ K. Thompson and N. Makri, Phys. Rev. E 59, R4729 (1999).

${ }^{26}$ H. Wang, M. Thoss, and W. H. Miller, J. Chem. Phys. 112, 47 (2000).

${ }^{27}$ K. Yamashita and W. H. Miller, J. Chem. Phys. 82, 5475 (1985).

${ }^{28}$ E. Rabani, G. Krilov, and B. J. Berne, J. Chem. Phys. 112, 2605 (2000).

${ }^{29}$ E. Sim, G. Krilov, and B. Berne, J. Phys. Chem. A 105, 2824 (2001).

${ }^{30}$ E. Gallicchio and B. J. Berne, J. Chem. Phys. 105, 7064 (1996).

${ }^{31}$ E. Gallicchio, S. A. Egorov, and B. J. Berne, J. Chem. Phys. 109, 7745 (1998).

${ }^{32}$ S. A. Egorov, E. Gallicchio, and B. J. Berne, J. Chem. Phys. 107, 9312 (1997).

${ }^{33}$ G. Krilov and B. J. Berne, J. Chem. Phys. 111, 9147 (1999).

${ }^{34}$ J. Cao and G. A. Voth, J. Chem. Phys. 100, 5093 (1994).

${ }^{35}$ J. Cao and G. A. Voth, J. Chem. Phys. 100, 5106 (1994).

${ }^{36}$ J. Cao and G. A. Voth, J. Chem. Phys. 101, 6157 (1994).

${ }^{37}$ J. Cao and G. A. Voth, J. Chem. Phys. 101, 6168 (1994).

${ }^{38}$ J. Cao and G. A. Voth, J. Chem. Phys. 101, 6184 (1994).

${ }^{39}$ S. Jang and G. A. Voth, J. Chem. Phys. 111, 2357 (1999).

${ }^{40}$ S. Jang and G. A. Voth, J. Chem. Phys. 111, 2371 (1999).

${ }^{41}$ A. Calhoun, M. Pavese, and G. A. Voth, Chem. Phys. Lett. 262, 415 (1996)

${ }^{42}$ U. W. Schmitt and G. A. Voth, J. Chem. Phys. 111, 9361 (1999).

${ }^{43}$ S. Jang, Y. Pak, and G. A. Voth, J. Phys. Chem. A 103, 10289 (1999).

${ }^{44}$ M. Pavese and G. A. Voth, Chem. Phys. Lett. 249, 231 (1996).

${ }^{45}$ K. Kinugawa, P. B. Moore, and M. L. Klein, J. Chem. Phys. 106, 1154 (1997).

${ }^{46}$ K. Kinugawa, P. B. Moore, and M. L. Klein, J. Chem. Phys. 109, 610 (1998).

${ }^{47}$ K. Kinugawa, Chem. Phys. Lett. 292, 454 (1998).

${ }^{48}$ M. Pavese, D. R. Berard, and G. A. Voth, Chem. Phys. Lett. 300, 93 (1999).

${ }^{49}$ S. Miura, S. Okazaki, and K. Kinugawa, J. Chem. Phys. 110, 4523 (1999).

${ }^{50}$ F. J. Bermejo et al., Phys. Rev. Lett. 84, 5359 (2000).

${ }^{51}$ D. R. Reichman, P.-N. Roy, S. Jang, and G. A. Voth, J. Chem. Phys. 113, 919 (2000).

${ }^{52}$ M. Hillery, R. F. O'Connell, M. O. Scully, and E. P. Wigner, Phys. Rep. 106, 121 (1984).

${ }^{53}$ R. Kubo, M. Toda, and N. Hashitsume, Statistical Physics IINonequilibrium Statistical Mechanics (Springer, Berlin, 1983).

${ }^{54}$ J. Poulsen, S. R. Keiding, and P. J. Rossky, Chem. Phys. Lett. 336, 488 (2001).

${ }^{55}$ B. Widom, J. Chem. Phys. 55, 44 (1971).

${ }^{56}$ J. L. Skinner and P. G. Wolynes, J. Chem. Phys. 69, 2143 (1978).

${ }^{57}$ D. Chandler, J. Chem. Phys. 68, 2959 (1978).

${ }^{58}$ J. A. Montgomrey, Jr., D. Chandler, and B. J. Berne, J. Chem. Phys. 70, 4056 (1979).

${ }^{59}$ N. Makri, Annu. Rev. Phys. Chem. 50, 167 (1999).

${ }^{60}$ J. Ankerhold and H. Grabert, Chem. Phys. 204, 27 (1996).

${ }^{61}$ B. B. Laird, J. Budimir, and J. L. Skinner, J. Chem. Phys. 94, 4391 (1991).

${ }^{62}$ E. Geva, E. Rosenman, and D. J. Tannor (unpublished).

${ }^{63}$ G. A. Voth, D. Chandler, and W. H. Miller, J. Phys. Chem. 93, 7009 (1989).

${ }^{64}$ W. H. Miller, J. Phys. Chem. A 102, 793 (1998).

${ }^{65}$ D. A. McQuarrie, Statistical Mechanics (Harper and Row, New York, 1976).

${ }^{66}$ H. Wang, X. Song, D. Chandler, and W. H. Miller, J. Chem. Phys. 110, 4828 (1999)

${ }^{67}$ S. Jang and G. A. Voth, J. Chem. Phys. 107, 9514 (1997).

${ }^{68}$ J. Cao, L. W. Ungar, and G. A. Voth, J. Chem. Phys. 104, 4189 (1995).

${ }^{69}$ D. Chandler, Introduction to Modern Statistical Mechanics (Oxford University Press, New York, 1987). 